\title{
IL-1/ dominates the promucin secretory cytokine profile in cystic fibrosis
}

\author{
Gang Chen, ${ }^{1}$ Ling Sun, ${ }^{1,2}$ Takafumi Kato, ${ }^{1}$ Kenichi Okuda, ${ }^{1}$ Mary B. Martino, ${ }^{1}$ Aiman Abzhanova, ${ }^{1}$ Jennifer M. Lin, ${ }^{1}$ \\ Rodney C. Gilmore, ${ }^{1}$ Bethany D. Batson, ${ }^{1}$ Yvonne K. O'Neal, ${ }^{1}$ Allison S. Volmer, ${ }^{1}$ Hong Dang, ${ }^{1}$ Yangmei Deng, ${ }^{1}$ Scott H. Randell, ${ }^{1}$ \\ Brian Button, ${ }^{1}$ Alessandra Livraghi-Butrico, ${ }^{1}$ Mehmet Kesimer, ${ }^{1}$ Carla M.P. Ribeiro, ${ }^{1}$ Wanda K. O'Neal, ${ }^{1}$ and Richard C. Boucher \\ 'Marsico Lung Institute and Cystic Fibrosis Research Center, University of North Carolina at Chapel Hill, Chapel Hill, North Carolina, USA. ²Research Center of Regeneration Medicine, West China Hospital, \\ Sichuan University, Chengdu, Sichuan Province, China.
}

\begin{abstract}
Cystic fibrosis (CF) lung disease is characterized by early and persistent mucus accumulation and neutrophilic inflammation in the distal airways. Identification of the factors in CF mucopurulent secretions that perpetuate CF mucoinflammation may provide strategies for novel CF pharmacotherapies. We show that IL-1 $\beta$, with IL-1 $\alpha$, dominated the mucin prosecretory activities of supernatants of airway mucopurulent secretions (SAMS). Like SAMS, IL-1 $\beta$ alone induced MUC5B and MUC5AC protein secretion and mucus hyperconcentration in CF human bronchial epithelial (HBE) cells. Mechanistically, IL-1 $\beta$ induced the sterile $\alpha$ motif-pointed domain containing ETS transcription factor (SPDEF) and downstream endoplasmic reticulum to nucleus signaling 2 (ERN2) to upregulate mucin gene expression. Increased mRNA levels of IL1B, SPDEF, and ERN2 were associated with increased MUC5B and MUC5AC expression in the distal airways of excised CF lungs. Administration of an IL-1 receptor antagonist (IL-1Ra) blocked SAMS-induced expression of mucins and proinflammatory mediators in CF HBE cells. In conclusion, IL- $1 \alpha$ and IL-1 $\beta$ are upstream components of a signaling pathway, including IL-1R1 and downstream SPDEF and ERN2, that generate a positive feedback cycle capable of producing persistent mucus hyperconcentration and IL-1 $\alpha$ and/or IL-1 $\beta$-mediated neutrophilic inflammation in the absence of infection in CF airways. Targeting this pathway therapeutically may ameliorate mucus obstruction and inflammation-induced structural damage in young CF children.
\end{abstract}

\section{Introduction}

Beginning soon after birth, cystic fibrosis (CF) lung disease is characterized by a heterogeneous, persistent mucoinflammatory environment. Abnormal airway epithelial ion transport predisposes the CF neonatal/childhood lung to mucoinflammatory responses thought to be triggered by viral infections and/or aspiration (1-3). Consistent with this notion, in the absence of recognizable bacterial infection, the bronchoalveolar lavage (BAL) of young children with $\mathrm{CF}$ contains mucus flakes containing both MUC5B and MUC5AC mucins accompanied by neutrophilic inflammation associated with increased IL- $1 \alpha$ and IL-1 $\beta$ concentrations $(3,4)$. Subsequently, CF lung disease is worsened by bacterial infection superimposed on this mucoinflammatory state $(5,6)$. Aggressive bacterial eradication fails to prevent developing bronchiectasis in CF children and ferrets (7-9), suggesting that chronic mucoinflammation is a critical factor resulting in structural damage. The pathways/interactions that trigger persistent mucoinflammatory responses in the absence or presence of bacterial infection in the CF lung remain unclear.

Related Commentary: p. 4089

Conflict of interest: The authors have declared that no conflict of interest exists. Copyright: @ 2019, American Society for Clinical Investigation.

Submitted: October 19, 2018; Accepted: July 18, 2019; Published: September 16, 2019. Reference information: J Clin Invest. 2019;129(10):4433-4450.

https://doi.org/10.1172/JCl125669.
Previous studies utilized CF airway secretions to identify the mechanisms of CF airway mucoinflammatory responses/disease (10-12). One recent study showed that supernatants of airway mucopurulent secretions (SAMS) administration to CF human bronchial epithelial (HBE) cell cultures increased both MUC5B and MUC5AC secretion without increase in ion/fluid secretion, producing abnormal mucus hyperconcentration (13). An unresolved question is the nature of the stimuli in SAMS that produced this combined MUC5AC/MUC5B response, i.e., bacterial versus host factors. IL- $1 \beta$ and IL- $1 \alpha$ are found in SAMS (13), BAL from CF infants (12), and neonatal Scnn1b-Tg mice (14), a model of CF mucoobstructive lung disease (15). These data, plus previous reports of the beneficial effects of reducing IL- $1 \alpha$ and IL-1 $\beta$ activity on mucus obstruction in Scnn1b-Tg mice (14), suggest that IL-1 $\beta$ and IL-1 $\alpha$ may be key mucin secretagogues in CF airway secretions. However, some in vitro studies reported that IL- $1 \beta$ induced MUC5AC but not MUC5B mRNA in primary HBE cells $(16,17)$, whereas other studies reported upregulation of MUC5B by IL-1 $\beta$ (18). Thus, the regulation of $M U C 5 B$ mRNA levels by IL- $1 \beta$ relevant to increased MUC5B levels in CF airway secretions is unresolved.

IL-1 $\beta$ and IL-1 $\alpha$ exert their activities through the IL-1 receptor, type I (IL-1R1) (19). A natural inhibitor of IL-1R1 (IL-1 receptor antagonist [IL-1Ra]) exists that modulates a variety of IL-1R1-related immune and inflammatory responses (20). Several potential downstream pathways may link IL-1R1 signaling to mucin hypersecretion in CF. One candidate is SAM-pointed domain containing ETS transcription factor (SPDEF), a transcription factor (TF) that 
regulates mucous cell differentiation and mucin production in health (21) and disease (22-24). A second candidate is endoplasmic reticulum to nucleus signaling 2 (ERN2), a transmembrane ER stress sensor. Like Spdef, Ern2 is expressed in mucin secretory cells and is required for baseline and TH2 inflammation-induced mucin production in the mouse airways (23-25). Increased mRNA levels of SPDEF and ERN2 are associated with activation of unfolded protein response (UPR) and increased MUC5B mRNAs in the distal airway epithelia in idiopathic pulmonary fibrosis (IPF) lungs (24). However, the roles of IL-1R1, SPDEF, and ERN2 in IL-1R1mediated regulation of both MUC5B and MUC5AC expression in mucoobstructive inflammatory diseases such as CF are unknown.

In the current study, we first investigated whether IL-1 $\alpha$ and/ or IL-1 $\beta$ activates both MUC5B and MUC5AC secretion in HBE cells, producing a MUC5B-dominated mucus after chronic exposure. Second, we used CRISPR/Cas9 technologies to determine whether the IL- $1 \alpha$ and/or IL-1 $\beta$ in SAMS are the major transcriptional regulators of both mucins and whether IL-1 $\beta$ is sufficient to raise $\mathrm{CF}$ airway mucus concentration. Third, we investigated whether IL- $1 \alpha$ and/or IL-1 $\beta$ signaling can induce mucin expression and secretion in vivo and whether these effects are mediated by IL-1R1 and SPDEF. Fourth, we tested for upregulation of IL1B and $M U C 5 B / M U C 5 A C$ mRNAs expression in excised CF lung distal airways using RNAscope and morphometry. Finally, we investigated IL-1R1 as a potential therapeutic target to disrupt the positive mucoinflammatory cycle centered on IL-1 $\alpha$ and/or IL-1 $\beta$ signaling. A combination of primary human epithelial culture, in vivo mouse models, and freshly excised human lung tissues were employed to investigate these questions.

\section{Results}

Relative effectiveness of SAMS components in regulation of mucin gene expression. We first screened the major inflammatory cytokines and interferons found in SAMS (13), including the TH17- and TH2-skewed cytokines reported in CF lungs (26), for MUC5B and MUC5AC mRNA regulatory activities (Supplemental Figure 1; supplemental material available online with this article; https:// doi.org/10.1172/JCI125669DS1). In these studies, non-CF HBE cells were exposed to 12 different recombinant human inflammatory mediators for 3 days, and their regulation of mucin gene expression was compared with SAMS. SAMS raised both MUC5AC and MUC5B mRNA levels. IL- $1 \alpha$, IL-1 $\beta$, TNF- $\alpha$, IL-17A, and IL-13 also raised MUC5AC mRNA expression. IL-1 $\beta$ and IL- $1 \alpha$ induced the greatest fold change of $M U C 5 B$ mRNAs $(71 \pm 16, P<0.001$ and $40 \pm 11, P=0.031$ respectively, mean \pm SEM; Supplemental Table 1 ), followed by IL-17F, TNF- $\alpha$, IL-17A, and IFN- $\beta$. In contrast, IL-13 and IFN- $\gamma$ suppressed MUC5B mRNA expression. These studies led us to focus our attention on IL-1 signaling as a major regulator of both MUC5B and MUC5AC production.

Exposure of $\mathrm{HBE}$ cells isolated from 14 independent non-CF donors to IL- $1 \alpha$, IL-1 $\beta$, or SAMS for 5 days produced increased MUC5B and MUC5AC mRNAs (Figure 1A). IL-13 (included as a control because its responses in HBE cells were well characterized) induced MUC5AC but inhibited MUC5B mRNAs, consistent with previous studies $(27,28)$. Because IL- $1 \alpha$ and IL- $1 \beta$ were the most active cytokines in stimulating mucin secretions, their absolute concentrations in SAMS were measured by ELISA. IL-1 $\beta$ was higher in concentration compared with IL-1 $\alpha$ (41.9 \pm 5.5 vs. $1.1 \pm$ $0.5 \mathrm{ng} / \mathrm{ml}, P<0.0001$, mean $\pm \mathrm{SEM}$ ) (Figure 1B).

To determine whether IL- $1 \alpha$ and IL-1 $\beta$ were the major mucin secretagogues in SAMS, a lentivirus vector expressing $I L-1 R 1$ gRNA and Cas9 (Supplemental Figure 2, A-C) was utilized to delete $I L-1 R 1$ in HBE cells. IL-1R1 CRISPR-modified HBE cells exhibited decreased MUC5B and MUC5AC mRNA expression in cells treated with PBS (baseline) compared with the control cells infected with lentivirus expressing EGFP gRNA/Cas9 (Figure 1C). Importantly, MUC5B and MUC5AC mRNA responses to SAMS exposure were markedly inhibited in $I L-1 R 1$ CRISPR-modified HBE cells (MUC5B decreased by $61 \% \pm 21 \%, P=0.013$ and $M U C 5 A C$ by $58 \%$ $\pm 31 \%, P=0.018$; mean \pm SD; Figure $1 \mathrm{C})$. Notably, in response to SAMS, the IL-1R1 CRISPR also suppressed mRNA expression of $S P D E F$ and epithelial derived proinflammatory mediators (IL8, Figure 1C; IL6 and CXCL1, Supplemental Figure 2D).

We next compared the histological changes in HBE cell cultures induced by IL- $1 \alpha$, IL-1 $\beta$, SAMS, and IL-13. IL- $1 \alpha$, and IL-1 $\beta$ (both at $10 \mathrm{ng} / \mathrm{ml}$ concentration in basolateral media) induced complex mucous cell metaplasia with an intraepithelial mucus accumulation associated with MUC5B and MUC5AC apical hypersecretion, which was similar to findings associated with induction by SAMS (Figure 1D). Induction of mucin genes was similar with apical versus basolateral IL-1 $\beta$ exposure (Supplemental Figure 3A). In contrast to IL- $1 \alpha$, IL-1 $\beta$ and SAMS, IL-13 promoted classic goblet cell differentiation and MUC5AC, but not MUC5B, intracellular expression (Figure 1D). IL-1 $\alpha$ and IL-1 $\beta$ induced MUC5B and MUC5AC protein secretion from HBE cells as detected by Western blot followed by densitometry semiquantification, while IL-13 did not significantly change the secretion of either mucin after a 7-day exposure (Supplemental Figure 3, B and C).

Interestingly, at low concentrations $(0.1 \mathrm{ng} / \mathrm{ml}), \mathrm{IL}-1 \beta$ induced classic goblet cell morphological changes, with both MUC5AC and MUC5B proteins present in mucin granules (Figure 2A). A higher concentration of $1 \mathrm{ng} / \mathrm{ml} \mathrm{IL-1 \beta}$ resulted in a shift in morphology with intraepithelial accumulation of mucus (Figure 2A). mRNA expression of MUC5B and MUC5AC was also IL-1 $\beta$ concentration dependent $\left(R^{2}=0.655, P<0.0001\right.$ vs. $R^{2}=0.198, P=0.022$, respectively) in the 0 to $1 \mathrm{ng} / \mathrm{ml}$ range (Figure 2, B and C). No significant differences in induction of MUC5B and MUC5AC mRNA were noted between $1 \mathrm{ng} / \mathrm{ml}$ and $10 \mathrm{ng} / \mathrm{ml}$ of IL-1 $\beta$ concentration (Supplemental Figure 3D).

Chronic IL-1 $1 \beta$ exposure leads to MUC5B-dominant mucin secretion. We next compared IL-1 $\beta$ versus IL-13 in regulation of mucin mRNA and protein expression utilizing a chronic exposure (5 week) protocol. IL-1 $\beta$ and IL-13 both induced formation of a thick apical mucus layer after 5 weeks (Figure 3A). Alcian blueperiodic acid Schiff-positive (AB-PAS-positive) glycoconjugates and histologically evident intraepithelial mucus accumulation were observed in IL-1 $\beta$-treated cells. Classic goblet cells filled with AB-PAS-positive material were evident in IL-13-treated cultures (Figure 3C). Under these conditions, IL-1 $\beta$ promoted synthesis and secretion of a MUC5B-dominant mucus, whereas IL-13 produced a MUC5AC-dominant mucus (Figure 3, B, D, and E).

$I L-1 \beta$ increases mucus concentrations on CF HBE cell surfaces. Previous studies reported that SAMS exposure increased the concentration of apical mucus of CF, but not non-CF, HBE cells 
A

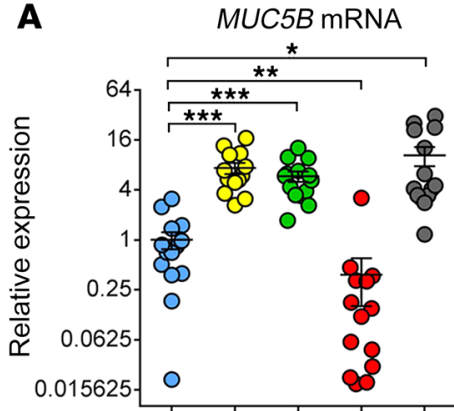

MUC5AC mRNA

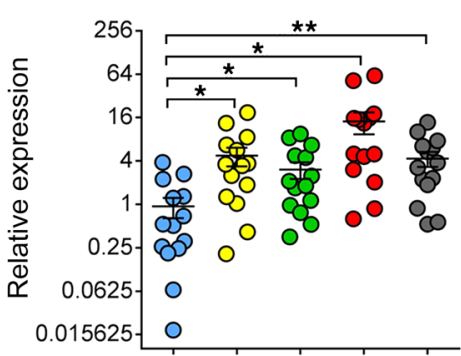

OControl

OIL-1 $\alpha$

OIL-1 $\beta$

OIL-13

OSAMS
C

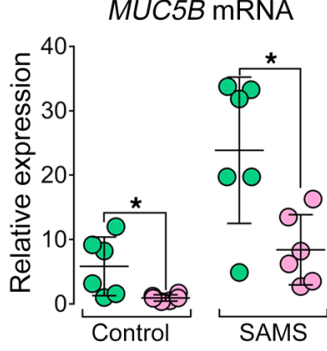

MUC5AC mRNA

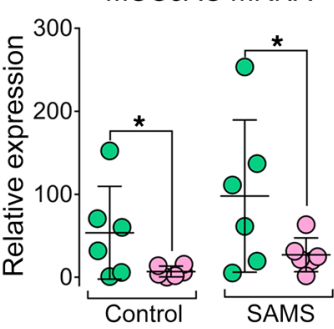

SPDEF mRNA

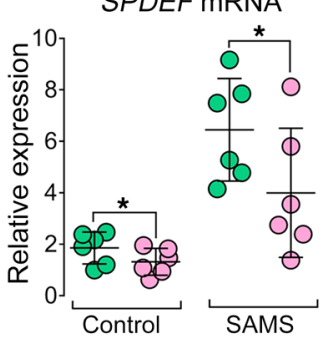

B IL- $1 \alpha / \beta$ concentration in SAMS

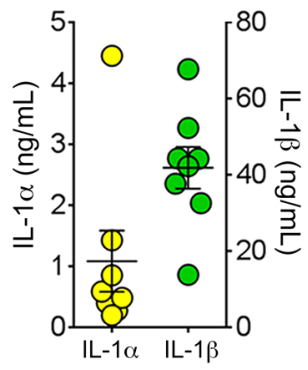

D

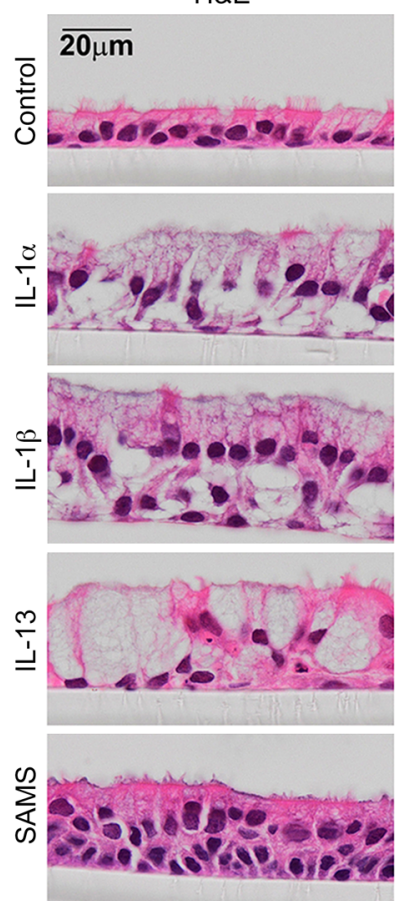

AB-PAS

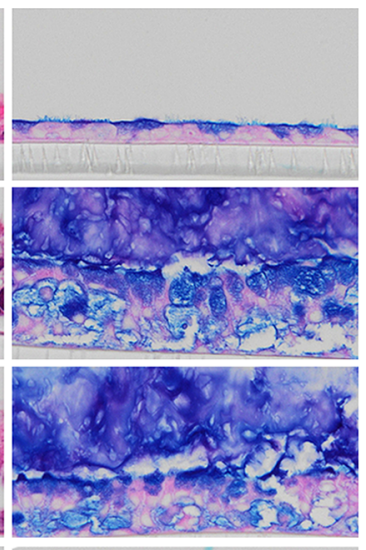

\author{
MUC5B
}
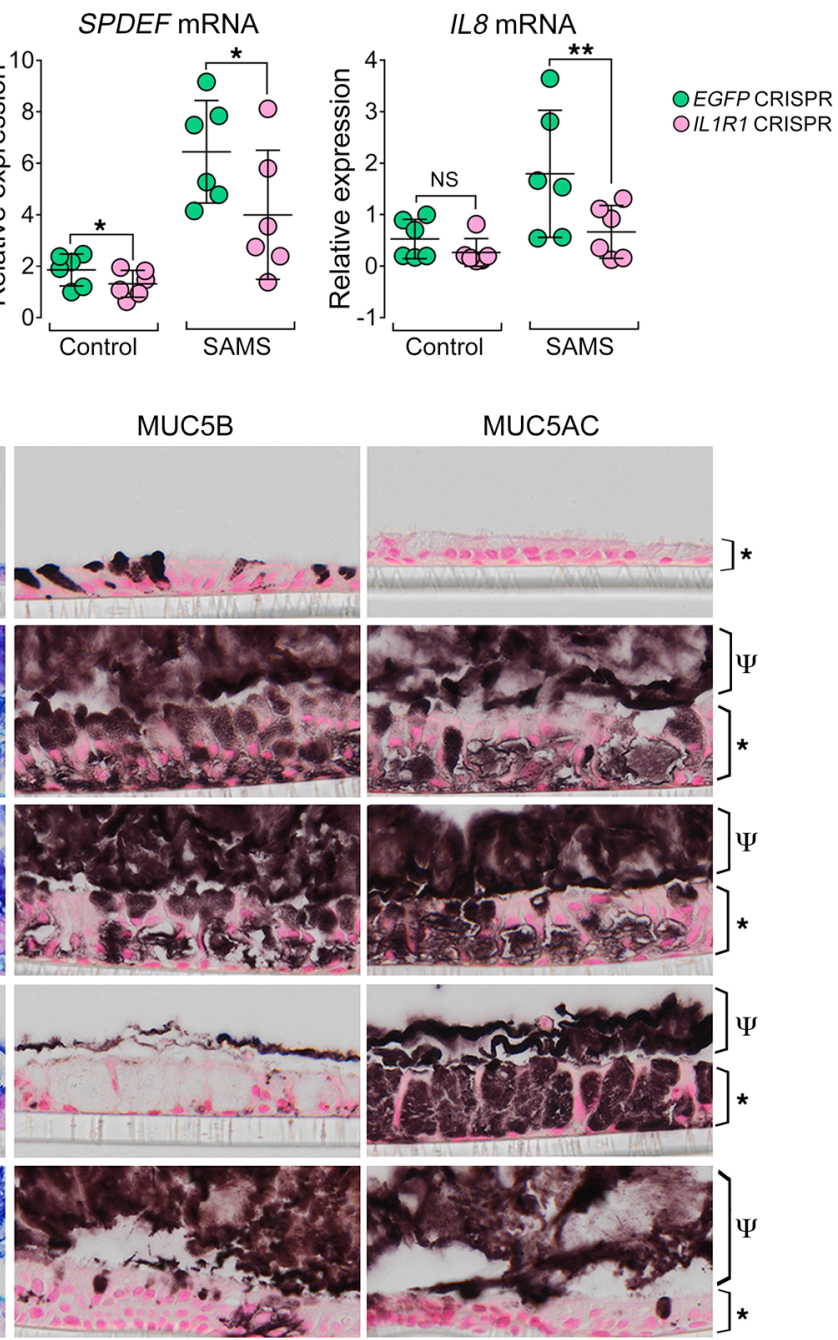

MUC5AC

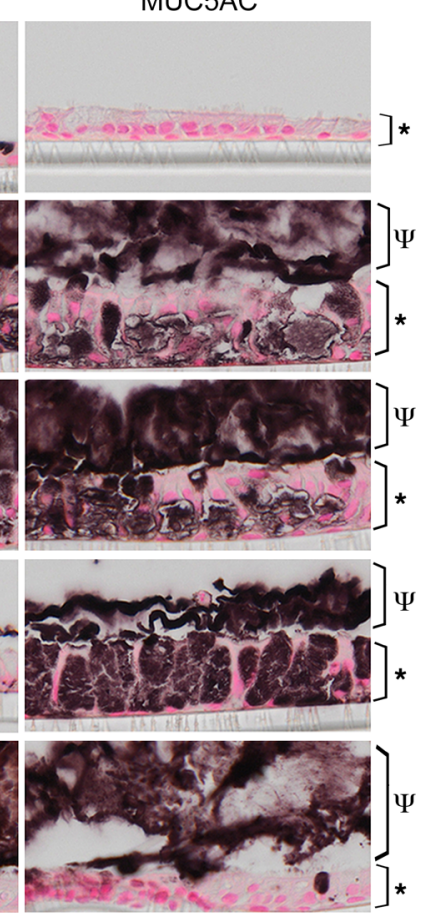

Figure 1. Mucin secretagogue activity of SAMS is mediated by IL-1 $\alpha$ and/or IL-1 $\beta$ via IL-1R1. (A) Expression of MUC5B and MUC5AC mRNAs in non-CF HBE cells as determined by TaqMan assays after exposure to control (PBS), IL-1 $\alpha$, IL-1 $\beta$, IL-13, or SAMS of CF lung for 5 days. IL-1 $\alpha$, IL-1 1 , and IL-13 were administered at $10 \mathrm{ng} / \mathrm{ml}$ in ALI media from basolateral side of the cells. Undiluted SAMS (50 $\mu$ l) was administered to the apical surface of HBE cells, where it was maintained until the cells were utilized for assays. Scatter plots present mean \pm SEM, with 1 culture of HBE cells obtained from 14 non-CF donors for each treatment condition. Data were analyzed with 1-way ANOVA followed by Dunnett's test. (B) IL-1 $\alpha$ and IL-1 $\beta$ protein concentration in SAMS was determined by ELISA. SAMS was collected from 8 CF lungs. Scatter plot presents mean \pm SEM. (C) Non-CF HBE cells were infected with lentiviruses expressing EGFP (control) or IL-1R1 CRISPR guide RNA and Cas9 protein. After ALI was cultured for 4 weeks, CRISPR/Cas9 targeted cells were treated with vehicle control (PBS) or SAMS for 3 days. MUC5B, MUC5AC, SPDEF, and IL8 mRNAs in HBE cells were quantitatively measured by TaqMan assays. Scatter plots present mean \pm SD. Data were derived from HBE cells from $n=6$ non-CF donors analyzed with 2-tailed paired $t$ test. ${ }^{*} P<0.05$; ${ }^{* *} P<0.01 ;{ }^{* *} P<0.001$. (D) Histological changes of HBE cells after cytokine treatment are shown by H\&E staining. Goblet cell differentiation and mucus production are shown by AB-PAS staining. Expression of MUC5B and MUC5AC protein is demonstrated by immunohistochemical staining. Micrographs present non-CF HBE cells of 3 donors. $\Psi$, mucus layer; *, epithelial cell layer. Scale bar: $20 \mu \mathrm{m}$. 
A

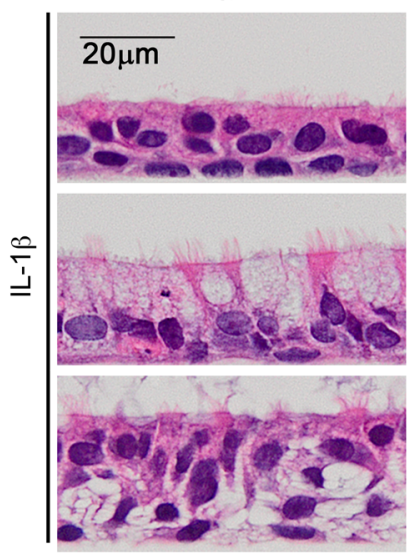

AB-PAS

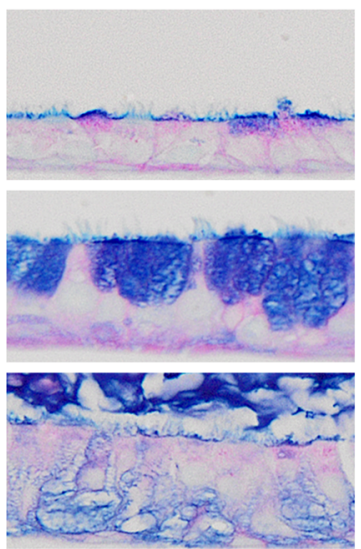

MUC5B

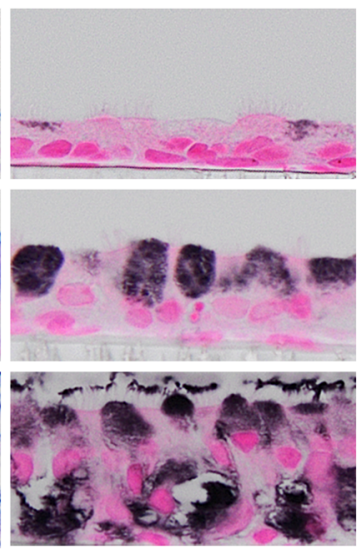

MUC5AC

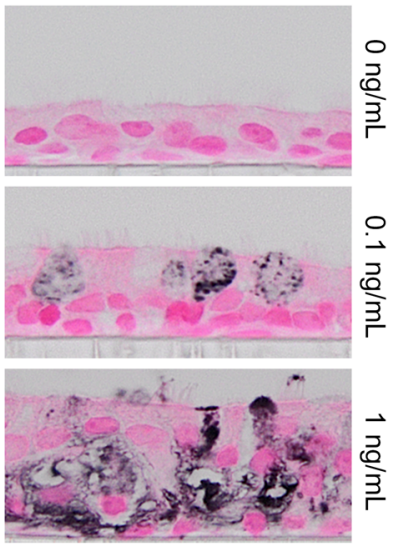

B

Correlation between MUC5B mRNA and IL-1 $\beta$ concentration

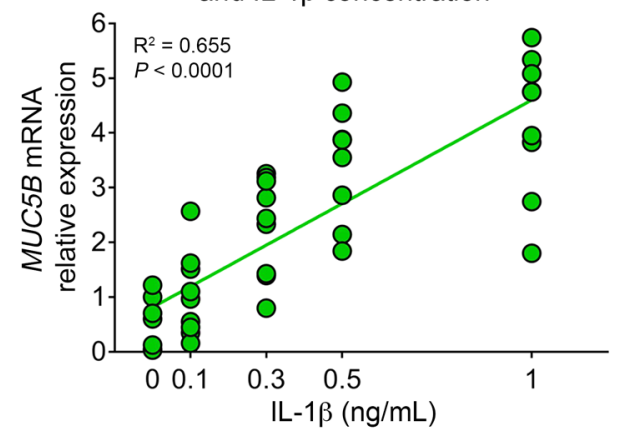

C
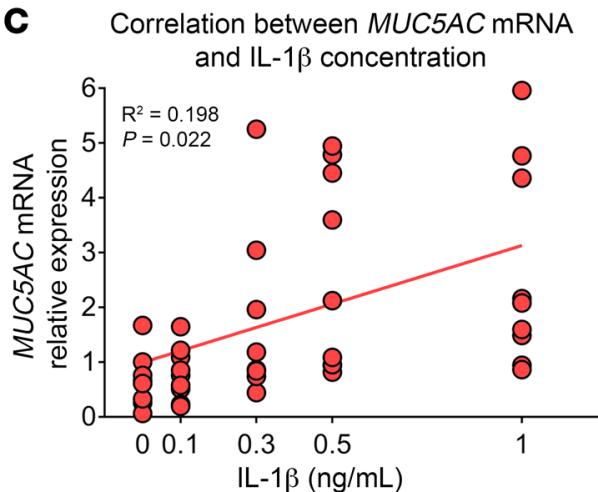

Figure 2. IL-1 $\beta$ induces goblet cell differentiation and MUC5B expression in a concentration-dependent manner. Non-CF HBE cells were treated with IL-1 $\beta$ at a gradient of concentrations of $0.1,0.3,0.5$, and $1 \mathrm{ng} / \mathrm{ml}$ from the basolateral side for 5 days. (A) Histological changes are shown by $\mathrm{H} \& \mathrm{E}$ staining and goblet cell differentiation by AB-PAS staining for treatment groups of $0,0.1$, and $1 \mathrm{ng} / \mathrm{ml}$ of IL-1 $\beta$. Expression of MUC5AC/MUC5B protein was identified by immunohistochemical staining. Micrographs are representative of HBE cells from $n=3$ donors in each treatment group. Scale bar: $20 \mu \mathrm{m}$. (B and $\mathbf{C})$ Linear regression plots show correlations between mRNA expression of MUC5B or MUC5AC and IL-1 $\beta$ concentration (non-CF HBE cells; $n=9$ donors).

(13). To determine whether IL-1 $\beta$ administration recapitulated this response, we evaluated epithelial morphology, MUC5B and MUC5AC protein and mRNA levels, and mucus wet-to-dry weight ratios (\% solids, a measurement of mucus concentration) after a 1-week exposure to IL-1 $\beta$ versus IL-13. IL-1 $\beta$ produced intraepithelial and extracellular AB-PAS positive material, and IL-13 produced the classic goblet cell morphology in CF HBE cells (Supplemental Figure 4A). In the conditionally reprogrammed cell (CRC) culture conditions (29-31) used here, baseline MUC5B and MUC5AC mRNAs and secreted protein and mucus percentage of solids were not different between $\mathrm{CF}$ and non-CF $\mathrm{HBE}$ cell cultures (Figure 4, A-C, and Supplemental Figure 4, B and C). In both non-CF and CF HBE cells, secreted MUC5B and MUC5AC protein and mRNA levels were significantly increased by IL-1 $\beta$, mimicking the effect of SAMS (Figure 4 , A and B, Supplemental Figure 4, B and C, and ref. 13). In contrast, IL-13 selectively induced MUC5AC protein secretion in CF cells, and IL-13 increased MUC5AC mRNA, but inhibited MUC5B mRNAs in both non-CF and CF cells (Figure 4, A and B, and Supplemental Figure 4, B and C). Notably, CF HBE cells, but not non-CF HBE cells, produced significantly higher apical mucus concentrations (percentage of solids) after IL-1 $\beta$ exposure compared with vehicle control (Figure 4C) regardless of whether the cells were initially expanded by CRC medium or the traditional bronchial epithelial cell growth medium (BEGM) (Supplemental Figure 5A). Thus, the CF HBE cells failed to mount a $\mathrm{Cl}^{-} /$fluid secretory response to hydrate newly secreted mucins after IL-1 $\beta$ stimulation (16).

Next, we evaluated the effects of IL-1 $\beta$, SAMS, and IL-13 on mRNA levels for CFTR and ANO1 (TMEM16A), whose fluid secretory functions would be predicted to govern mucus concentrations (32). Although there was considerable variability in responses among human samples, 1-week exposure to IL-1 $\beta$ and SAMS moderately but significantly induced CFTR mRNA, while IL-13 marginally increased CFTR mRNA, in both non-CF and CF HBE cells (Supplemental Figure 5B). In contrast, TMEM16A mRNA was induced by IL-13, but not by IL-1 $\beta$ or SAMS, in both non-CF and CF HBE cells (Supplemental Figure 5C).

$I L-1 \alpha$ and IL-1 $\beta$ induce mucin gene expression and secretion in vivo. To determine whether human in vitro culture findings exhibit relevance to in vivo conditions, wild-type (WT) adult C57BL/6J mice were exposed via intratracheal instillation to SAMS or purified recombinant murine cytokines IL-1 $\alpha$, IL-1 $\beta$, and IL-13. SAMS and all 3 cytokines induced goblet cell metaplasia and increased Muc5b and Muc5ac protein and mRNA expression in the epithelia lining the conducting airways (Figure 5A). Consistent with these findings, Muc5b and Muc5ac protein secretion in BAL were increased in the 
A

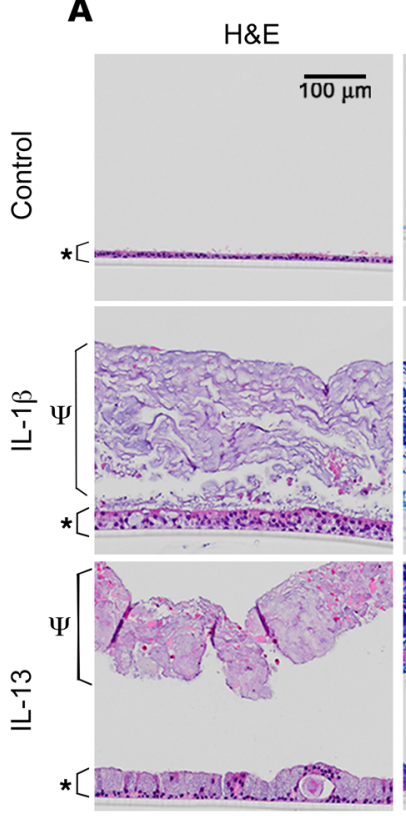

AB-PAS

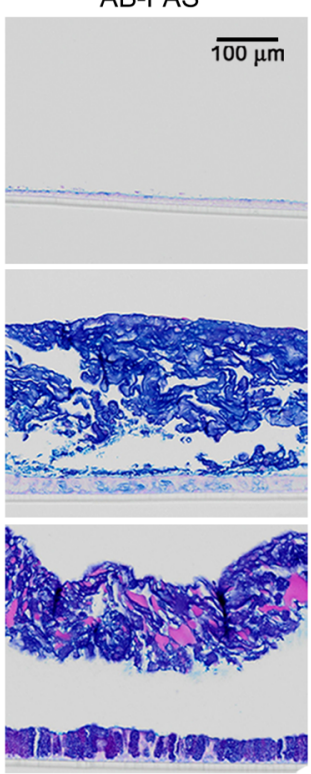

B

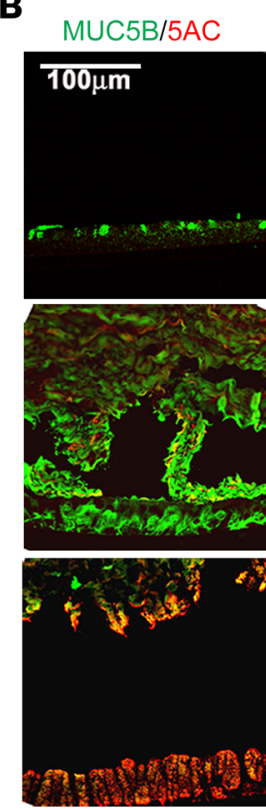

c

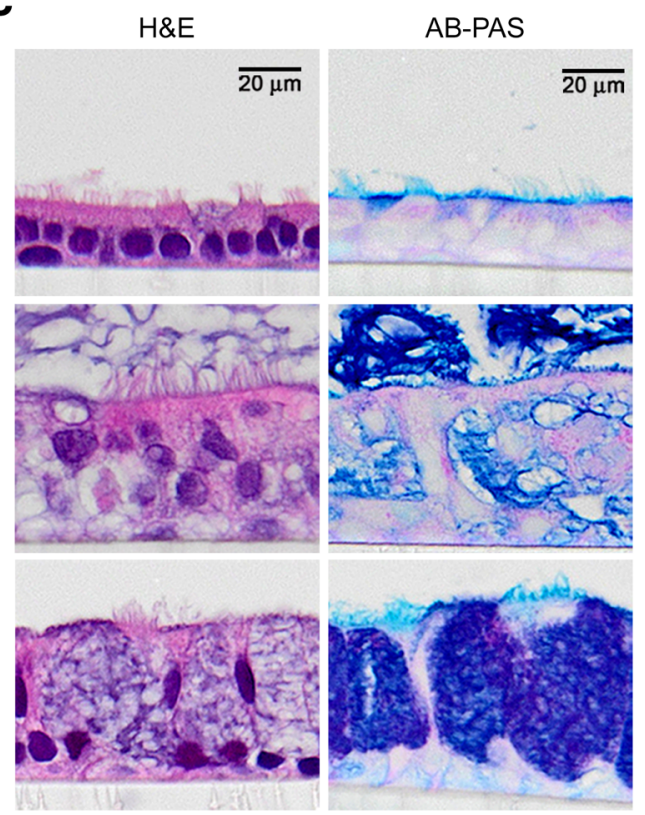

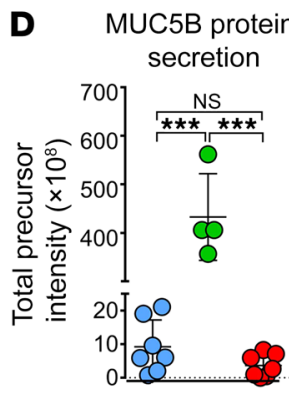
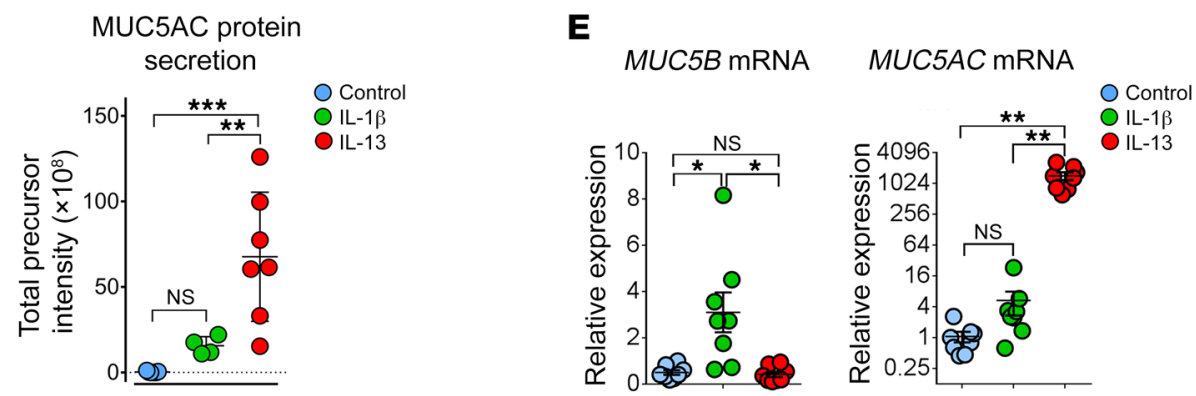

Figure 3. Chronic IL-1 $\beta$ exposure induces MUC5B-dominated mucin production. Non-CF HBE cells were exposed to vehicle control (PBS), IL-1 $1 \beta$, or IL-13 $(10 \mathrm{ng} / \mathrm{ml}$, from basolateral side in media) for 5 weeks without washing off the apical secretions. (A) Morphology of mucus layers, epithelial cell layers, and goblet cell differentiation are shown by H\&E and AB-PAS staining. $\Psi$, mucus layer; ${ }^{*}$, epithelial cell layer. (B) Expression of MUC5B and MUC5AC in HBE cells after chronic IL-1 $\beta$ and IL-13 exposure was determined by dual-immunofluorescent staining. (C) High-power view of mucus layers, epithelial cell layers, and goblet cell differentiation showed in A. All micrographs are representatives of HBE cells from $n=3$ donors in each treatment group. (D) Apical secretions of HBE cells after 5-week chronic exposure were subjected to trypsin digestion and analyzed with liquid chromatography-tandem mass spectrometry. MUC5B and MUC5AC proteins were identified and their quantities shown by total precursor intensities. HBE cells tested in control, IL-1 1 , and IL-13 exposure were collected from $n=8,4$, and 8 non-CF donor lungs, respectively. Scatter plots present mean \pm SD. (E) mRNA expression of MUC5B and MUC5AC was measured by TaqMan assays in the HBE cells exposed to control (PBS), IL-1ß, and IL-13 for 5 weeks. Data are represented as mean \pm SEM. Non-CF HBE cells from $n=8$ donor lungs were tested in each treatment group. Data were analyzed with 1-way ANOVA followed by Tukey's test (D and E). ${ }^{*} P<0.05 ;{ }^{* *} P<0.01 ;{ }^{* *} P<0.001$, compared with control groups. Scale bars: $100 \mu \mathrm{m}$ (A and B); $20 \mu \mathrm{m}$ (C).

mice administered with all 3 cytokines (Figure 5B), and mucin mRNAs were increased by all treatments in the whole-lung homogenates (Figure 5, C and D). All 3 cytokine treatments also induced expression of the goblet cell marker Clca1 (Gob5) (Supplemental Figure 6A), consistent with the histological findings.

Thus, except for the finding that Muc5b mRNA and protein were increased in IL-13-treated mice, the murine in vivo studies essentially replicated the in vitro results of HBE cells. To explore the discrepant IL-13 responses of Muc5b expression in mice in vivo, we evaluated the expression of pulmonary cytokines after IL-13 and IL- $1 \alpha$ and/or IL-1 $\beta$ administration. Interestingly, IL-13 cytokine exposure induced endogenous Illa and Il1b mRNAs, and IL-1 $\alpha$ and L-1 $1 \beta$ exposure induced endogenous Il1b and Il17a mRNAs in the whole lung (Supplemental Figure 6B), providing the mechanisms for Muc5b upregulation in mice in vivo.
$I L-1 R 1$ is required for $I L-1 \beta$-induced mucin and SPDEF expression in vivo. To test whether Il1r1 is required for IL-1 $\beta$-induced mucin gene expression in vivo, Il1r1-deficient $\left(\right.$ Illr1 $\left.^{-1-}\right)$ and WT $\left(I l 1 \mathrm{r}^{+++}\right)$mice were exposed via intratracheal instillation to recombinant murine IL-1 $\beta$ cytokine. Muc5b and Muc5ac mRNA expression in response to IL-1 $\beta$ were blunted in the lungs of Illr1 ${ }^{-/}$mice (Figure 6A). Spdef mRNA, which was increased after IL-1 $\beta$ administration in WT mice, was also inhibited after both saline and IL-1 $\beta$ exposure in Il1r1-deficient mice (Figure 6A). Induction of Muc5b, Muc5ac, and Spdef mRNAs by IL-1 $\alpha$ and IL-1 $\beta$ cytokine exposure was also suppressed in fully differentiated Illr1 ${ }^{-1}$ mouse tracheal epithelial cells (mTEC) (Figure 6B). In contrast, IL-13 induction of Muc5ac and Spdef mRNAs expression were preserved in $I l 1 r 1^{-1}$ mTECs (Figure 6B). Notably, in contrast to HBE cells, Muc5b was not downregulated by IL-13 in WT or 
A
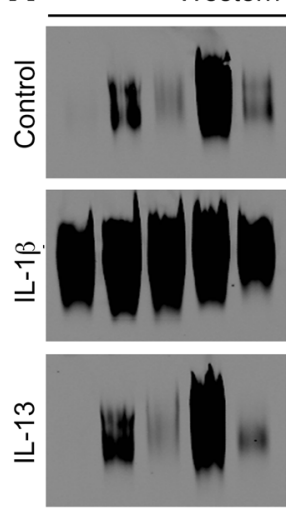

Non-CF HBE cells
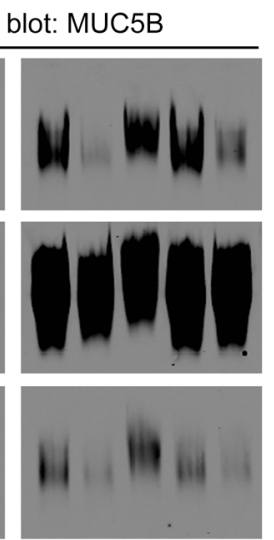

CF HBE cells

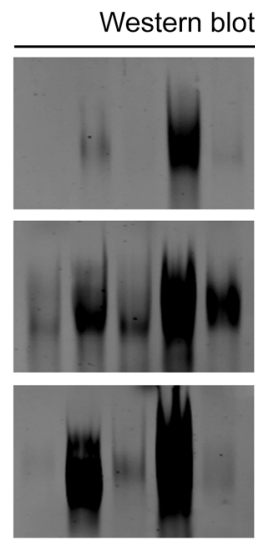

Non-CF HBE cells
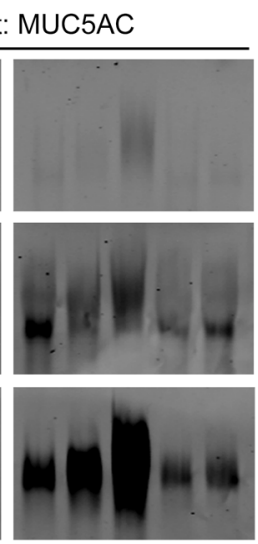

CF HBE cells
$\mathbf{B}$

\section{MUC5B protein in apical} secretions
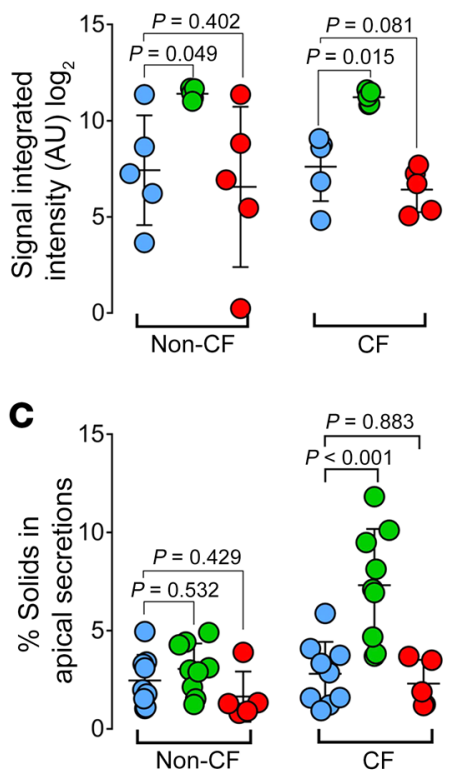

MUC5AC protein in apical secretions

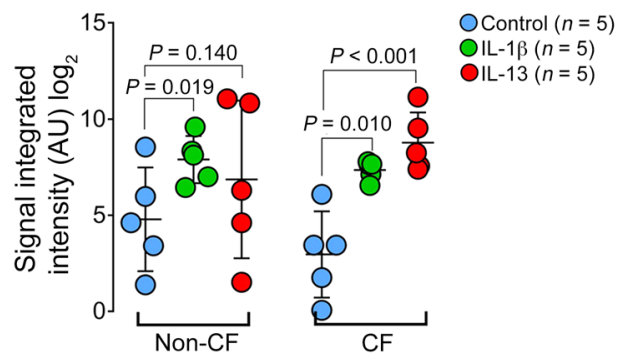

Figure 4. IL-1 $\beta$, but not IL-13, increases mucus percentage of solids in the apical secretions of CF HBE cells. Fully differentiated non-CF and CF HBE cells were exposed to control (PBS), IL-1 $\beta$, or IL-13 (10 ng/ml from basolateral side in media) for 1 week. (A) Apical secretions from non-CF and CF HBE cells were collected by washing the apical surface of HBE cells with PBS containing $10 \mathrm{mM}$ DL-dithiothreitol (DTT) to remove mucus. Secreted MUC5B and MUC5AC proteins were identified by mucin agarose gel Western blot. HBE cells from $n=$ 5 non-CF donors and $n=5$ CF donors were used for each treatment condition. (B) MUC5B and MUC5AC protein content shown in $\mathbf{A}$ was semiquantified with Licor Odyssey software. Scatter plots represent mean \pm SD. Data were analyzed with 1-way ANOVA followed by Dunnett's test. (C) The percentage of mucus solids content, an index of hydration of apical secretions, was measured from HBE cells after 1 week of treatment with control, IL-1 $\beta$ (cells from $n=$ 9 non-CF and $n=9$ CF donors), or IL-13 (cells from $n=5$ non-CF and $n=5$ CF donors). Data were analyzed with 1-way ANOVA followed by Dunnett's test.
$I L-1 R 1^{-/}$mTECs, as confirmed with multiple sets of probes (Figure 6B and Supplemental Figure 7A).

IL-1R1 CRISPR-modified HBE cells also exhibited decreased $M U C 5 B, M U C 5 A C$, and IL 8 mRNAs at baseline and lost IL-1 $\beta$ induction of MUC5B, MUC5AC, SPDEF, IL8, IL6, and CXCL1 mRNAs in comparison with EGFP (control) CRISPR-targeted cells (Figure 6C and Supplemental Figure 7B).

Spdef mediates $I L-1 \beta$-induced mucin production. To gain further insights into the IL-1 $\beta$-induced signaling pathway, RNA-Seq was performed in normal HBE cells cultured with or without IL-1 $\beta$ for 24 hours. SPDEF was one of the most increased TFs (Supplemental Figure 8A). In further studies, SPDEF mRNA was found to be induced by IL-1 $\beta$ and SAMS, although to a lesser extent compared with the well-known SPDEF inducer IL-13 (22), in both non-CF and CF HBE cells (Supplemental Figure 8, B and C).

We next evaluated whether SPDEF, paralleling its role in $\mathrm{TH} 2$-dominated inflammation $(23,33)$, is required for IL-1 $\beta$-triggered mucus production by airway epithelia in vivo. WT (Spdeft/+) and Spdef-deficient $\left(S p d e f^{-/}\right)$mice were administered with saline or recombinant murine IL-1 $\beta$ cytokine via intratracheal instilla- tion. IL-1 $\beta$ induced Muc5ac, Muc5b protein/mRNA, and Spdef mRNA in WT mice (Figure 7, A-E). Absence of Spdef reduced goblet cell differentiation and suppressed Muc5b and Muc5ac protein/mRNA expression/secretion in the BAL both at baseline and after IL-1 $\beta$ (Figure 7, A-F, and Supplemental Figure 9). Spdefregulated, mucin-pathway genes Foxa3 and Agr2 mRNAs (23) were also decreased in Spdef-deficient mice (Figure 7F).

$S P D E F$ induces ERN2 to regulate mucin gene expression in airway epithelial cells. The UPR gene ERN2 is selectively expressed in secretory epithelia lining the intestine and respiratory tracts. ERN2 functions overlap with those of Spdef $(24,25)$ to induce mucin gene expression, suggesting involvement in the same signaling cascade. To delineate a possible SPDEF-ERN2 hierarchy in IL-1 $\beta$-induced mucus production in vivo, RNAscope and TaqMan assays were performed to measure expression of Ern2 and a closely related isoform, Ern1 (34), in Spdef-deficient vs. WT mice. In naive adult Spdef-deficient mice, Ern2, but not Ern1, mRNA was absent in submucosal glands (SMGs) and superficial epithelia lining the proximal intrapulmonary airways, where Spdef is normally expressed (Supplemental Figure 10). Ern2, but not Ern1, mRNA 
A
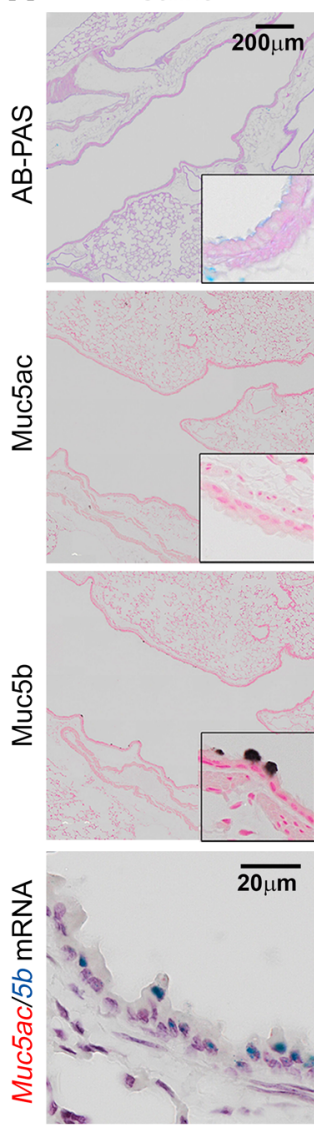

$\mathrm{IL}-1 \alpha$
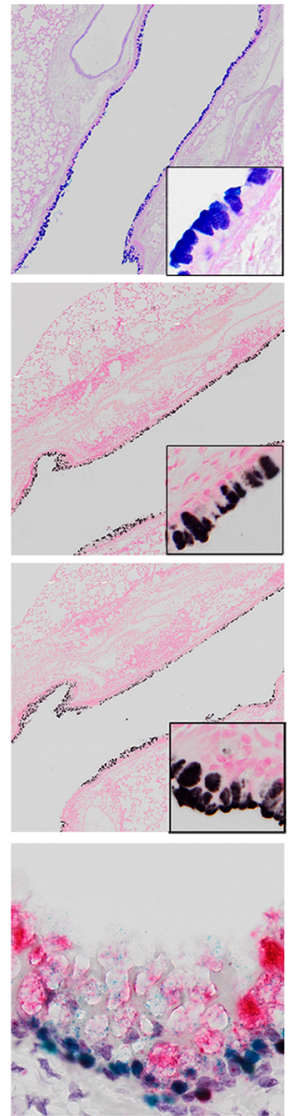

IL-1 $\beta$
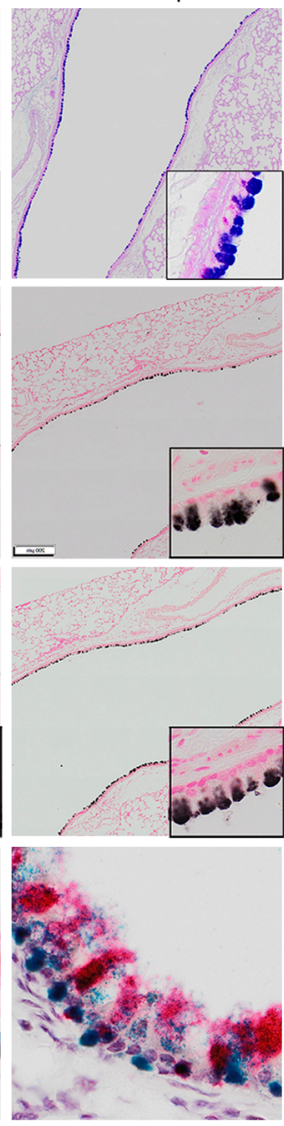

IL-13
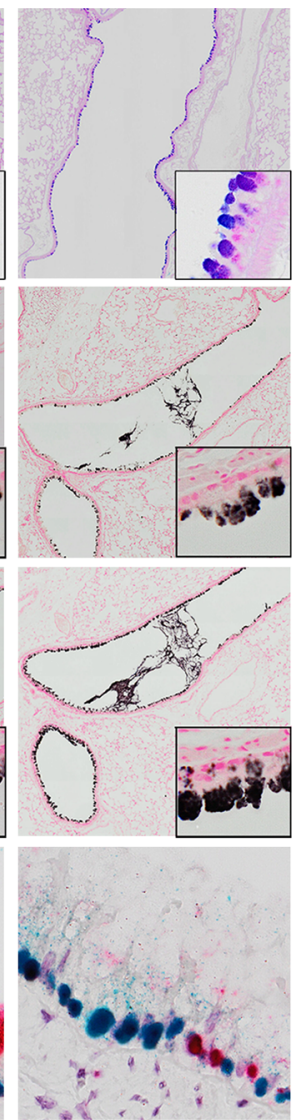

SAMS
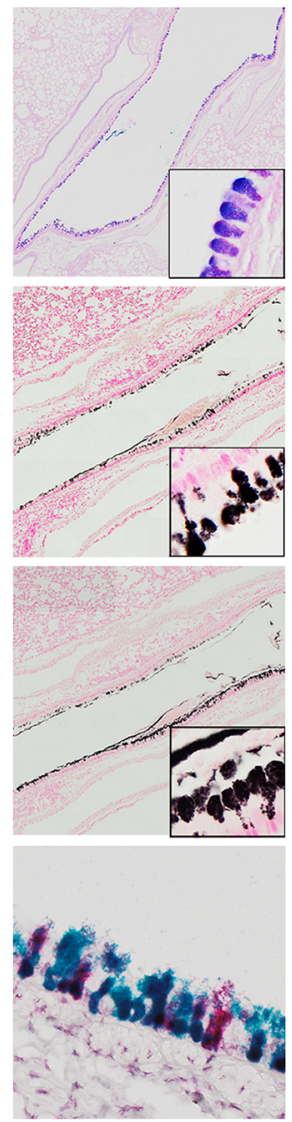

B

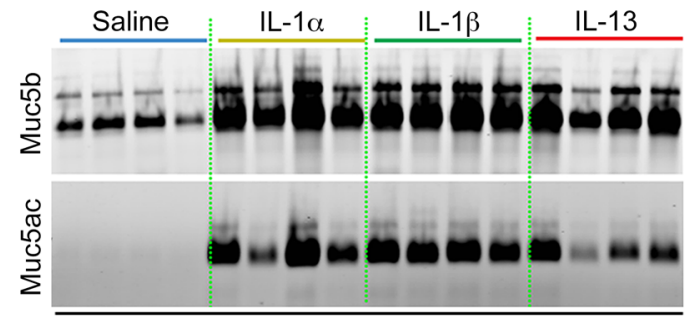

Secreted mucin in BAL
C

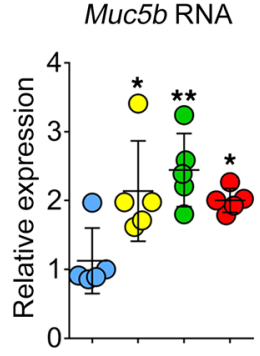

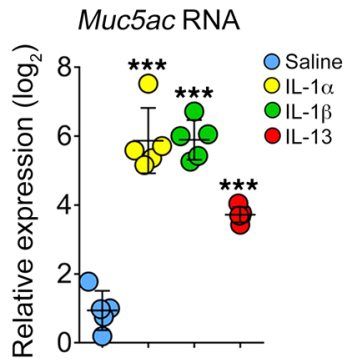

D Muc5b Muc5ac

mRNA mRNA

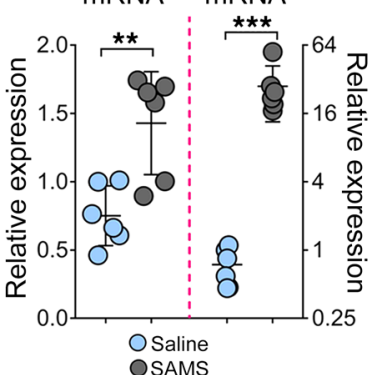

Figure 5. SAMS, IL-1 $\alpha$, IL-1 $\beta$, and IL-13 induce goblet cell differentiation and mucus production in vivo. (A) WT adult (6 weeks) female C57BL/6] mice were exposed to sterile saline and murine recombinant IL-1 $\alpha$, IL-1 $\beta$, IL-13, or SAMS via intratracheal instillation. Goblet cell differentiation and Muc5b and Muc5ac protein/mRNA expression in conducting airway epithelia were identified by AB-PAS and immunohistochemical staining and RNAscope (Muc5ac/Muc5b) duplex assays. Inserts show high-power view of airway epithelia. Micrographs are representatives of lung histology of $n=3$ mice/treatment group. Micrographs of AB-PAS, Muc5ac, and Muc5b immunohistochemical staining were taken at the same magnification. Scale bars: $200 \mu \mathrm{m}$ (top three rows); $20 \mu \mathrm{m}$ (bottom row). Original magnification, $\times 40$ (insets). (B) Secreted Muc5b and Muc5ac proteins in BAL were examined by mucin agarose gel Western blot ( $n=4$ mice/treatment group). (C) mRNA expression of Muc5b and Muc5ac in the whole lung was quantitatively measured by TaqMan assays after saline, IL-1 $\alpha$, IL-1 $\beta$, and IL-13 ( $n=5$ mice/treatment group). (D) Muc $5 b$ and Muc5ac mRNAs in the whole lung were quantitatively measured in mice exposed to saline and SAMS ( $n=6$ mice/group). Scatter plots present data as mean \pm SD; data were analyzed with 1-way ANOVA followed by Dunnett's test (C) and 2-tailed, unpaired $t$ test $(\mathbf{D}) .{ }^{*} P<0.05 ;{ }^{* *} P<0.01 ;{ }^{* *} P<0.001$, compared with saline groups.

expression was also substantially reduced in airway epithelia of Spdef-deficient mice after saline (control) and IL-1 $\beta$ exposure (Figure 8, A and B). Consistent with the notion that Spdef regulates the Ern2 cascade in vivo, overexpression of a FLAG-Spdef lentivirus in normal HBE cells (Supplemental Figure 11A) induced ERN2 mRNA, MUC5B, and MUC5AC mRNA/protein expression/ secretion and Spdef-regulated genes FOXA3 and AGR2, but not ERN1 mRNAs (Figure 8, C-E, and Supplemental Figure 11, B-D). Further, inhibition of SPDEF expression by transfection of SPDEF siRNA suppressed SPDEF, ERN2, MUC5B, and MUC5AC, but not ERN1 mRNAs expression, in an immortalized mucus-producing HBE cell line UNCN3T (ref. 35 and Figure 8F). 


\section{A}

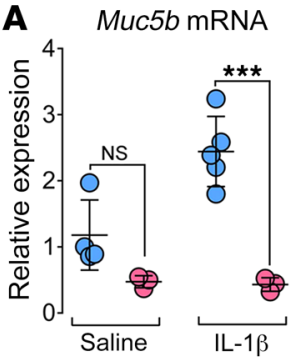

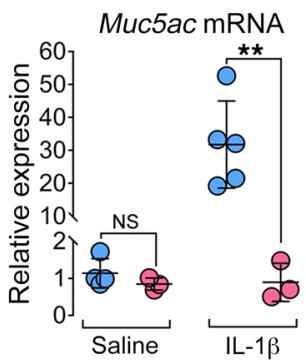

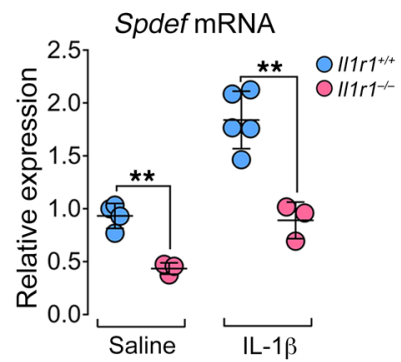

B Muc5b mRNA
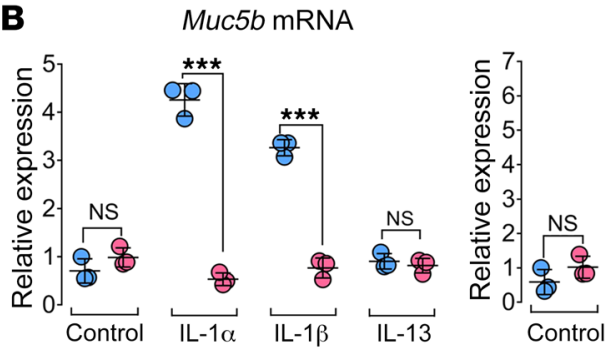

Muc5ac mRNA
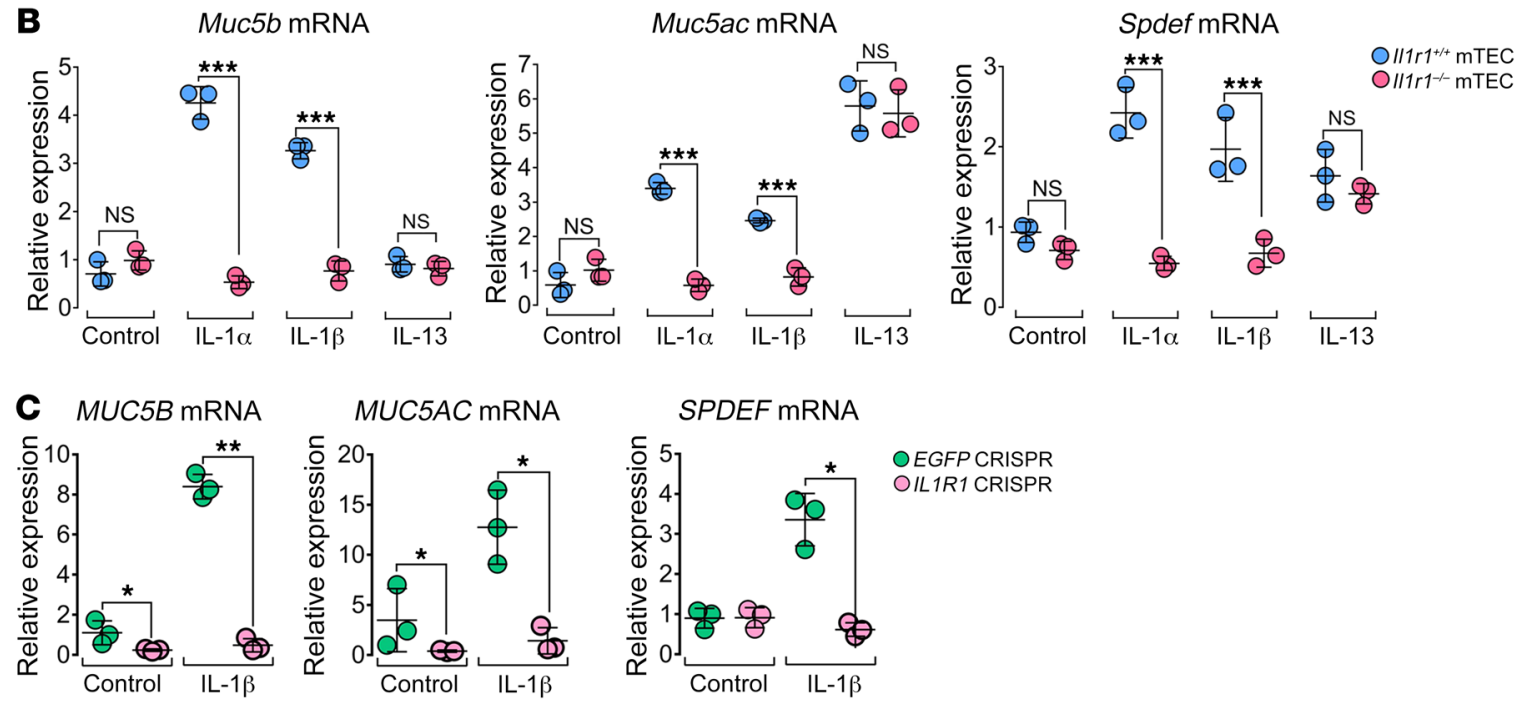

OEGFP CRISPR

OIL1R1 CRISPR

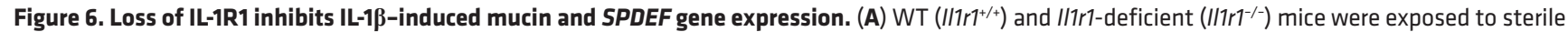
saline or murine recombinant IL-1 $\beta$ cytokine via intratracheal instillation, and mRNA expression of Muc5b, Muc5ac, and Spdef in the whole lung was quantitatively determined by TaqMan assays. $n=3-5$ mice/treatment/genotype. (B) mTECs isolated from WT and $/ 17 r^{-/-}$mice were cultured under air-liquid interface conditions for 3 weeks to allow full differentiation prior to exposure with vehicle control (PBS) or murine recombinant cytokines IL-1 $\alpha$, IL-1 $\beta$, and IL-13 for 1 week from basolateral media (all at $10 \mathrm{ng} / \mathrm{ml}$ ). mRNA expression of Muc5b, Muc5ac, and Spdef was quantitatively measured by TaqMan assays with $n=3$ independent mTEC cultures/treatment/genotype. Scatter plots in $\mathbf{A}$ and $\mathbf{B}$ present data as mean $\pm \mathrm{SD}$, and data were analyzed with 2-tailed unpaired $t$ test. (C) Non-CF HBE cells were infected with lentiviruses expressing EGFP (control CRISPR) and IL-1R1-CRISPR guide RNAs and Cas9 protein. After culturing at air-liquid interface for 4 weeks, CRISPR/Cas9 targeted cells were treated with vehicle control (PBS) or IL-1 $\beta$ for 3 days. MUC5B, MUC5AC, and SPDEF mRNAs in HBE cells were quantitatively measured by TaqMan assays. Scatter plots present data as mean $\pm \mathrm{SD}$, and data were analyzed with 2-tailed paired $t$ test with HBE cells from $n=3$ donor lungs. ${ }^{*} P<0.05$; ${ }^{* *} P<0.01$; ${ }^{* *} P<0.001$, compared with $11111^{+/+}$groups (A and B) or EGFP CRISPR groups (C).

Expression of IL1B, SPDEF, and ERN2 mRNAs is associated with mucin gene expression in CF lung tissue. RNAscope assays were performed to localize mRNA expression of MUC5AC/MUC5B, IL-1B/ $I L 1 A, S P D E F$, and ERN1/ERN2 in proximal bronchi transitioning to terminal bronchioles (Figure 9, A-D) in non-CF and CF lungs. $M U C 5 B$ mRNA was detected in proximal airway superficial epithelium of non-CF lungs, with a gradual reduction of expression in distal-to-terminal airways (Figure 9A). In the CF lungs, MUC5B mRNA expression was greater in the distal airways than in non-CF lungs, and MUC5B mRNA was also detected in the terminal airways. MUC5AC mRNA was weakly expressed in proximal airways of nonCF lungs, but strongly expressed in proximal bronchi in CF lungs. In distal-to-terminal airways, MUC5AC mRNA was rarely detected in control subjects, but readily detectable in CF airways (Figure 9A).

Neither IL1B nor IL1A mRNA was readily detected in nonCF lungs (Figure 9B). In CF lungs, IL1B mRNA was observed in the cells trapped in luminal mucus plugs and occasionally in the cells present in the airway epithelia (Figure 9B). IL1A mRNA was weakly detected in CF cells in the epithelial layer and sporadically detected in the cells trapped in the airway lumen (Figure 9B and Supplemental Figure 12). SPDEF signals were detected throughout the airway, but at increased levels in $\mathrm{CF}$ compared with non-CF (Figure 9C). ERN2 mRNA expression was also greatly increased in $\mathrm{CF}$ compared with non-CF in proximal and distal airway epithelia (Figure 9D). The MUC5B and MUC5AC mRNA expression patterns overlapped with those of SPDEF and ERN2 in CF lungs, supporting roles for SPDEF and ERN2 in mediating IL-1 $\beta$-induced mucin gene expression.

The overall visual expression patterns observed for these genes were confirmed by both morphometric (focusing on distal airways) and quantitative reverse-transcriptase PCR (RT-PCR) analyses. First, morphometric analyses were performed to quantitate the expression of MUC5B/MUC5AC and IL1B/IL1A mRNAs in CF versus non-CF distal airways (luminal diameter $\leq 1.5 \mathrm{~mm}$ ) (Supplemental Figure 12). MUC5B mRNA expression was significantly higher $(P=0.020)$, and MUC5AC mRNA showed a trend toward higher expression $(P=0.062)$ in the distal airway epithelia of $\mathrm{CF}$ versus non-CF subjects (Figure 10A). IL1B mRNA expression was 


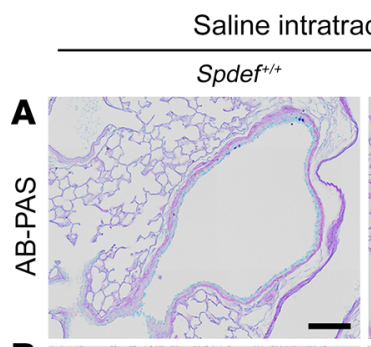

acheal instillation
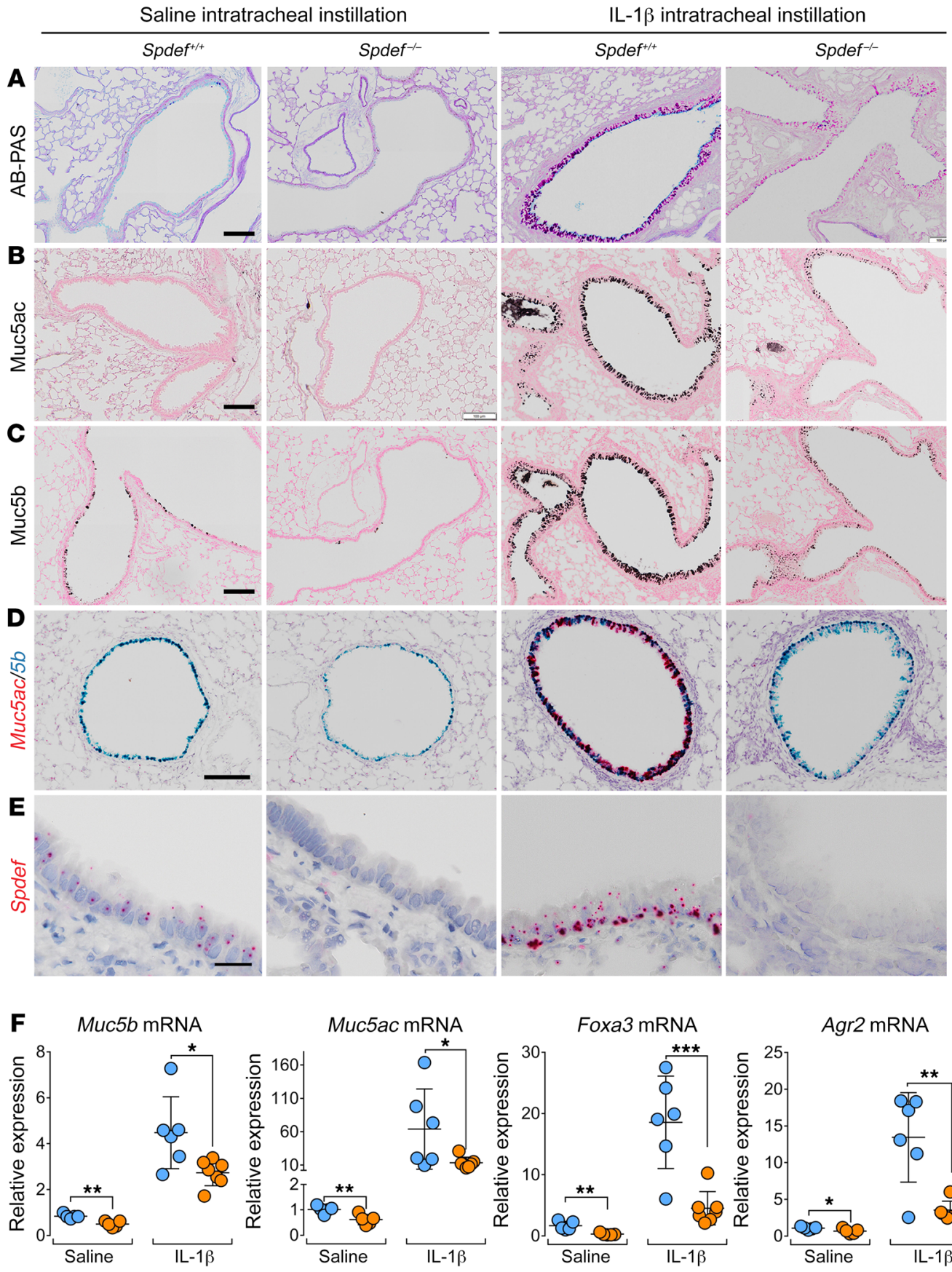

Figure 7. Spdef is required for IL-1 $\beta$-induced goblet cell differentiation and mucin production in vivo. WT (Spdef $\left.f^{+/}\right)$and Spdefdeficient $\left(\right.$ Spde $^{-/}$) 6-week-old mice were exposed to sterile saline or murine recombinant IL-1 $\beta$ cytokine via intratracheal instillation. Coblet cell differentiation was examined by AB-PAS staining (A), and Muc5ac and Muc5b protein/ mRNA expression in the conducting airway epithelia was assessed by immunohistochemical staining (B and C) and RNAscope duplex assays (D). (E) Spdef mRNA expression in the conducting airway epithelia was identified by RNAscope red assays. (A-E) Micrographs are representative of $n=3$ mice/treatment/ genotype. Scale bars: $100 \mu \mathrm{m}$ (A-D); $20 \mu \mathrm{m}$ (E). (F) mRNA expression of Muc5ac, Muc5b, Foxa3, and Agr2 from the whole lung was quantitatively measured by TaqMan assays In the Spdef ${ }^{+/+}$mouse group, $n=5$ and $n=6$ mice were treated with saline and IL-1 $\beta$, respectively. In the Spdef ${ }^{-/}$mouse group, $n=5$ and $n=7$ mice were treated with saline and IL-1 $1 \beta$, respectively. Scatter plots present data as mean $\pm S D$, and data were analyzed with 2-tailed unpaired $t$ test. ${ }^{*} P<0.05$; ${ }^{* *} P<$ 0.01 ; ${ }^{* *} P<0.001$, compared with saline groups. higher $(P=0.014)$ in the cells trapped in the distal airway lumens, with a trend toward higher expression $(P=0.062)$, in the distal airway epithelia, in $C F$ versus non-CF subjects. In contrast, $I L-1 A$ mRNA was not significantly different between $\mathrm{CF}$ and non-CF subjects in either compartment (Figure 10, B and C). Consistent with RNAscope data, quantitative RT-PCR conducted on mRNAs from airway epithelial cells freshly isolated from $\mathrm{CF}$ compared with non-CF/nonsmoker donors demonstrated increases in SPDEF, ERN2, MUC5B, and MUC5AC mRNAs in CF compared with nonCF subjects (Figure 10D). Further, SPDEF-regulated genes FOXA3 mRNAs (23) were also increased in CF versus non-CF freshly isolated HBE cells (Supplemental Figure 13A). Importantly, SPDEF mRNA expression correlated with ERN2 mRNA levels, and both were associated with increased $M U C 5 B$ and MUC5AC mRNAs expression in CF airway cells (Supplemental Figure 13, B-D).

$I L-1 R$ antagonist suppresses expression of SAMS-induced mucins and proinflammatory mediators. Administration of IL-1Ra concurrently to both apical and basolateral surfaces of $\mathrm{HBE}$ cells inhibited SAMS-induced MUC5B and MUC5AC mucin gene expression (Figure 11, A and B). This effect was associated with inhibition of SPDEF and the mucin chaperone protein AGR2 (36) mRNA expression in both non-CF and CF HBE cells (Figure 11, C and D). IL-1Ra inhibited SAMS-induced ERN2 upregulation in non-CF, but not CF, HBE cells (Figure 11E). These data suggest the presence of other pathways that may regulate ERN2 mRNA expression in CF epithelia. Gene expression of proinflammatory mediators, 


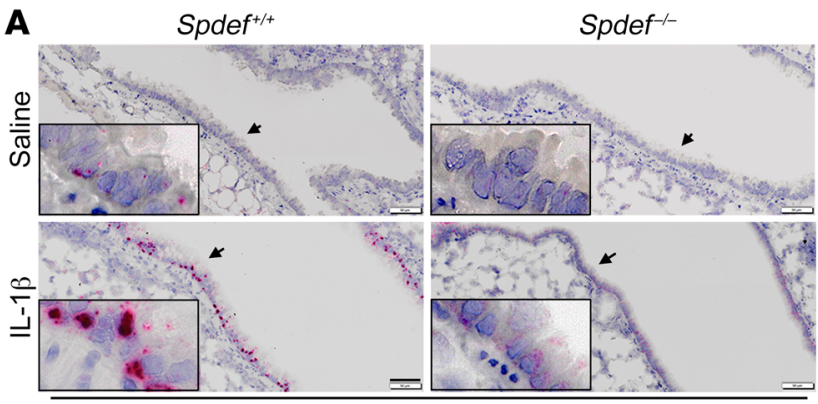

Ern2 RNAscope

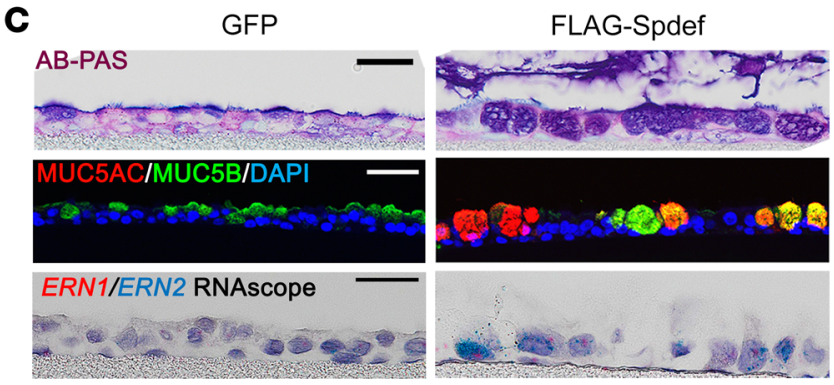

B

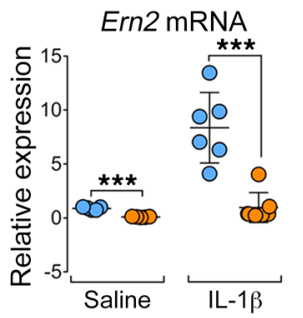

D

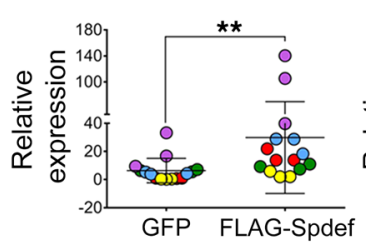

E

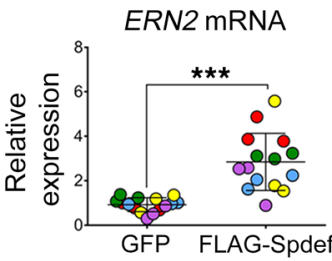

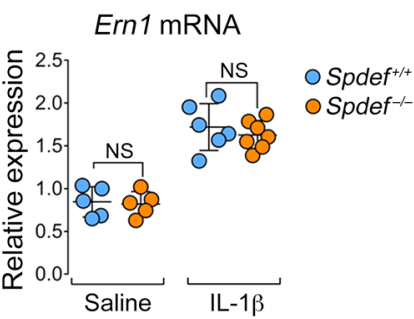

MUC5AC MRNA
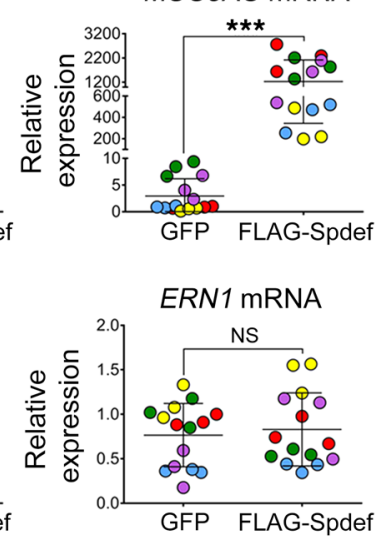

F
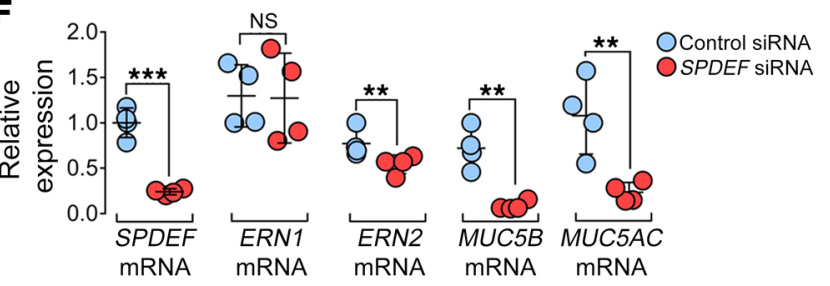

Figure 8. SPDEF regulates ERN2 and mucin production in vivo and in vitro. (A) WT (Spdef $f^{/+}$) and Spdef-deficient (Spdef $f^{-/}$) 6-week-old mice were exposed to sterile saline or murine recombinant IL-1 $\beta$ via intratracheal instillation. Ern2 mRNA in conducting airways was detected by RNAscope assay. Micrographs are representative of $n=3$ mice/treatment/genotype. Arrows point to regions shown in inserts. (B) Ern2 and Ern1 mRNAs in whole lung were quantitatively measured by TaqMan assays. The scatter plots present data as mean \pm SD; data were analyzed with 2-tailed unpaired $t$ test. For Spdeft/+ mice, $n=5$ and $n=6$ mice were administered saline and IL-1 $\beta$, respectively. For Spdef $f^{-1}$ mice, $n=5$ and $n=7$ mice were administered saline and IL-1 $\beta$, respectively. (C) Non-CF HBE cells were infected with lentiviruses expressing GFP (control) or FLAG-Spdef fusion protein and cultured under ALI conditions for 1 week. Coblet cell differentiation and MUC5B/MUC5AC protein expression were revealed by AB-PAS and dual-immunofluorescent staining, respectively. (D) MUC5B and MUC5AC mRNA levels were quantitatively measured in GFP vs FLAG-Spdef-transduced cultures by TaqMan assays. ERN1/ERN2 mRNA expression was identified by RNAscope duplex assay and quantitatively determined by TaqMan assay (C and E). Data were analyzed with 2-way ANOVA followed by Šidák's correction. Non-CF HBE cells from $n=5$ donors ( $n=3$ independent cultures/donor) were used for lentiviruses infection, and the same donor cells infected with GFP or FLAG-Spdef viruses were labeled with color-matching dots. (F) Immortalized HBE cells (UNCN3T) were transfected with negative control or SPDEF-specific siRNA, and SPDEF, ERN1, ERN2, MUC5B, and MUC5AC mRNAs were quantitatively measured after 48 hours. Scatter plots present data as mean \pm SD with $n=4$ independent cell cultures, and data were analyzed by 2-tailed, unpaired $t$ test. ${ }^{*} P<0.05$; ${ }^{* *} P<0.01$; ${ }^{* *} P<$ 0.001 , compared with saline (B), GFP ( $\mathbf{D}$ and E), and control siRNA (F) groups. Scale bars: $50 \mu \mathrm{m}$ (A); $20 \mu \mathrm{m}$ (C). Original magnification, $\times 60$ (insets).

e.g., IL8, IL6, and CXCL1, and innate host defense molecules, e.g., $\beta$-defensin 2 (DEFB4B) and lactotransferrin ( $L T F)$ (37), was also decreased by recombinant IL-1Ra at baseline and/or after SAMS administration in non-CF and CF HBE cells (Figure 11, F-H, and Supplemental Figure 14).

\section{Discussion}

$\mathrm{CF}$ presents as a progressive respiratory disease characterized by airway mucus obstruction, chronic neutrophilic inflammationassociated airway destruction, i.e., bronchiectasis, and bacterial infection (38). Neonatal/early CF childhood lung disease is characterized by a bacterially sterile, mucoinflammatory state that includes adhesion of mucus plaques/plugs to airway surfaces with local mucus/epithelial hypoxia (39). It appears likely that the next stage of disease reflects infection of hypoxic mucus by anaerobic bacteria aspirated from the oral cavity into the lung $(6,40)$. Subsequently, there is acquisition of classic CF bacterial pathogens, e.g., Staphylococcus and Pseudomonas. Acquisition of these bacteria is associated with an acceleration of the rate of loss of lung function (41). There are multiple aspects of this paradigm that remain unclear. First, the pathogenesis of CF airway mucus stasis/obstruction has been assigned to either raised mucus concentrations or reduced $\mathrm{pH}$ (4244). Second, the site of initial abnormal mucus production, i.e., large airway SMGs versus small airway superficial epithelia, is debated (3, 45). Finally, it is unclear how a "vicious" mucoinflammatory cycle that perpetuates and accelerates CF lung disease is initiated. 
A

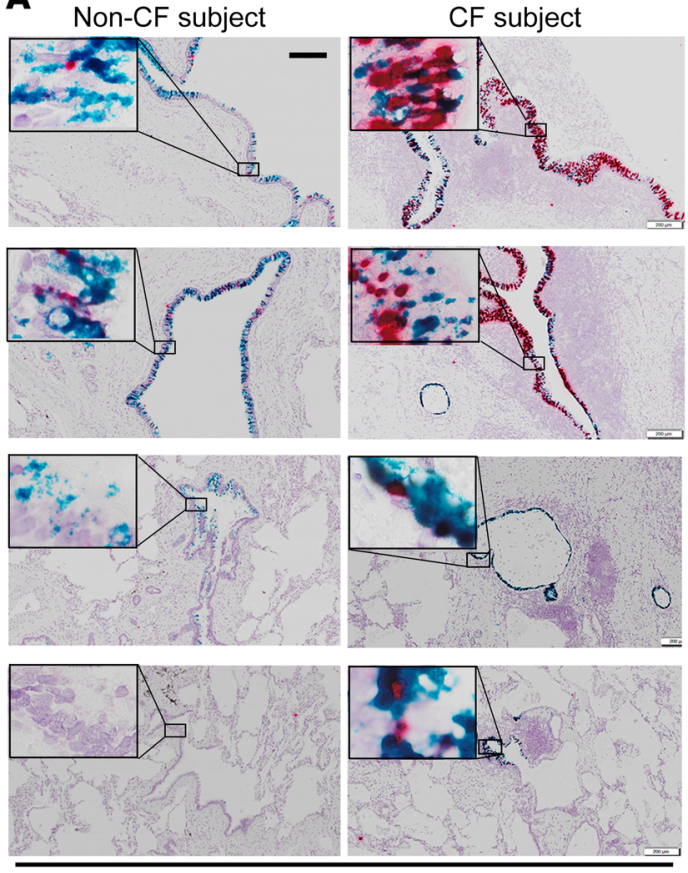

MUC5AC/MUC5B mRNA
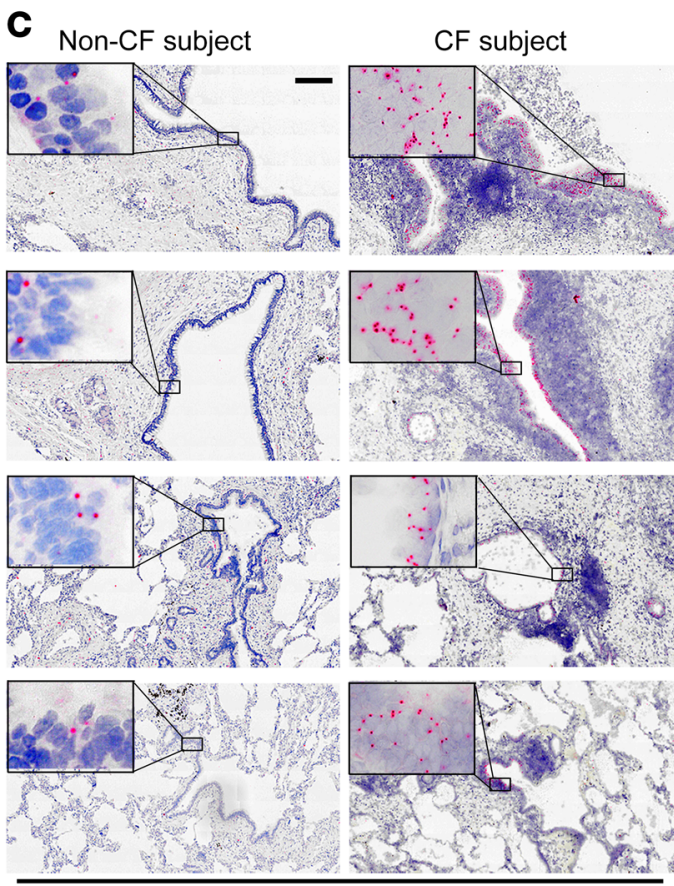

SPDEF MRNA
B
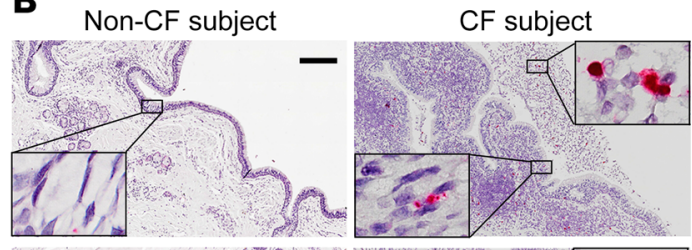

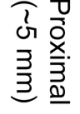
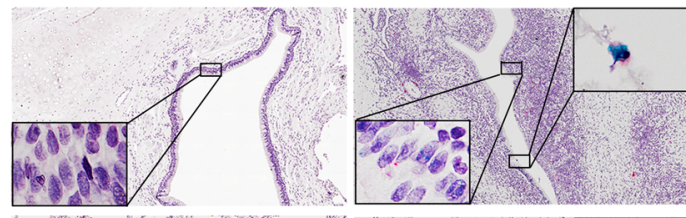

$\widehat{N} \overline{\frac{7}{\sigma}}$

$+\frac{1}{3}$

콜

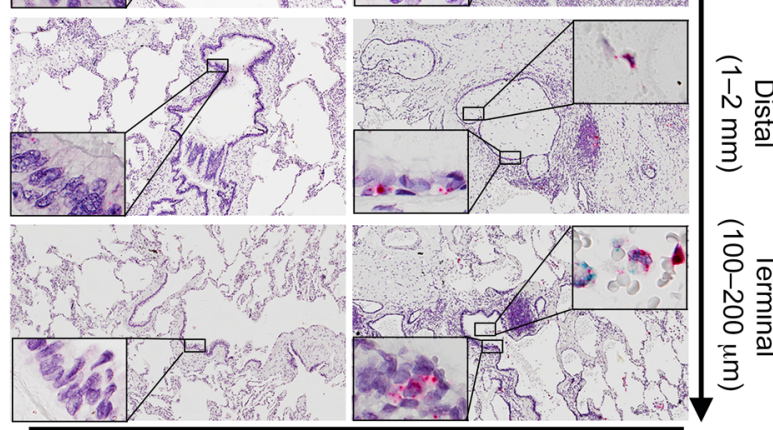

IL1B/IL1A mRNA

D Non-CF subject
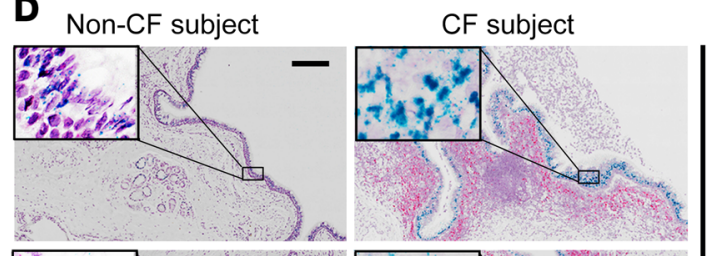

ioํㅜㅇ

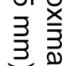
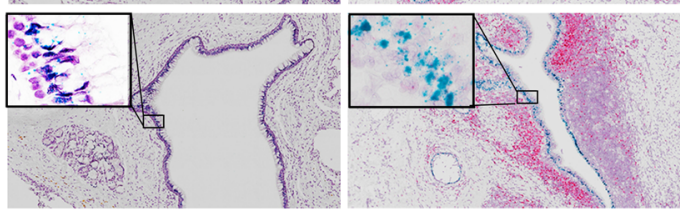

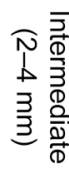
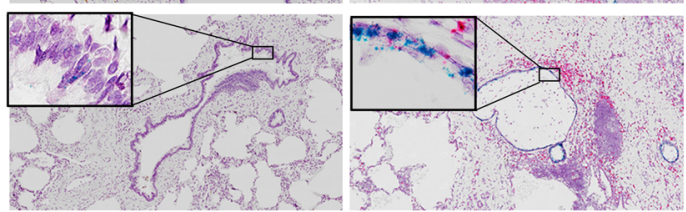

ב

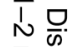

$3 \stackrel{3}{3} \stackrel{\frac{9}{ \pm}}{\underline{D}}$

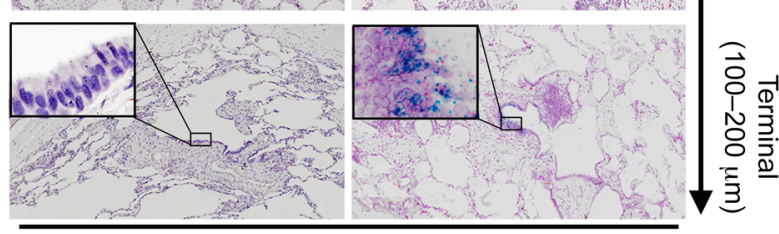

ERN1/ERN2 mRNA

Figure 9. Expression of IL1B, SPDEF, and ERN2 mRNAs is associated with MUC5B mRNA in proximal to terminal airway epithelia in CF. Expression of MUC5AC, MUC5B, IL1B, IL1A, SPDEF, ERN1, and ERN2 mRNAs was detected by RNA in situ hybridization in non-CF (control) and CF lung subjects. The representative histological sections contain airways ranging from the proximal (bronchial) airways (the regions containing SMG; the airway luminal diameter is around $5 \mathrm{~mm}$ ) to intermediate $(2-4 \mathrm{~mm})$ and distal $(1-2 \mathrm{~mm})$, and further to terminal regions $(\leq 200 \mu \mathrm{m})$ in each panel. Detection of expression of these genes was performed on the matched sequential sections for each assay. MUC5AC/MUC5B (A) and IL1B/IL1A (B) mRNAs were detected by RNAscope duplex assays. (C) Detection of SPDEF mRNA expression by Basescope assays. (D) ERN1/ERN2 mRNA expression was detected by RNAscope duplex assays. In B, the inserts at bottom left corners show high-power view of the selected areas of airway tissue from both non-CF and CF subjects, and the inserts at the upper right corners show high-power views of the selected area containing the cells trapped in the luminal mucus plugs in CF lung tissue. In $\mathbf{A}, \mathbf{C}$, and $\mathbf{D}$, inserts show high-power view of rectangle-selected areas on airway tissues. Micrographs are representative of RNAscope assays performed with $n=4$ non-CF and $n=3$ CF lungs. Scale bars: $200 \mu \mathrm{m}$. Original magnification, $\times 60$ (insets). 

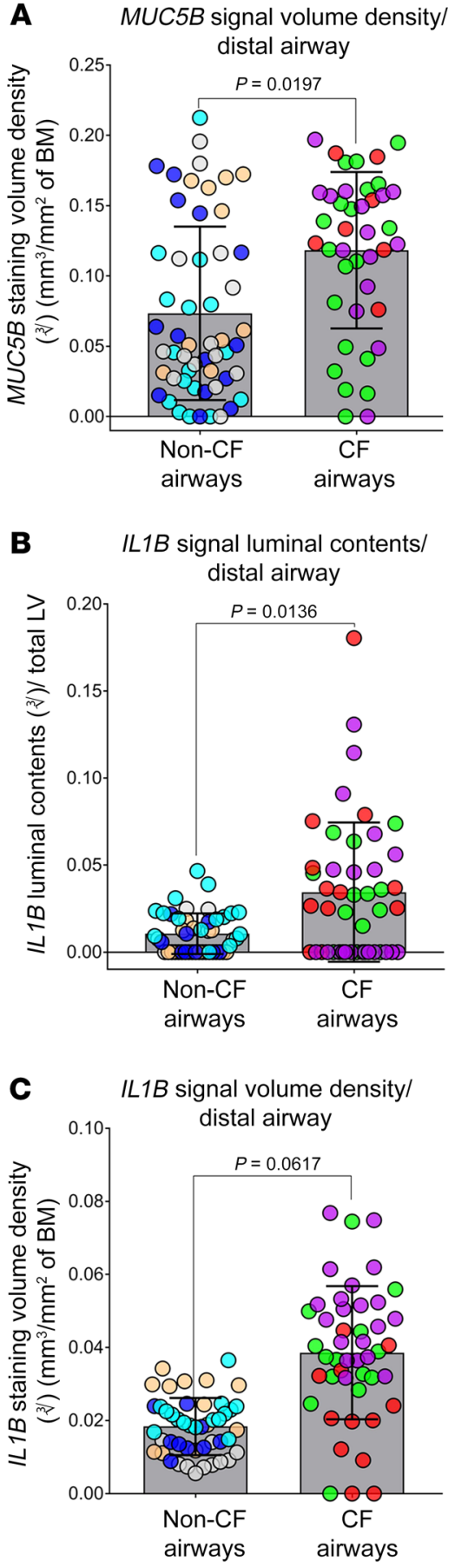
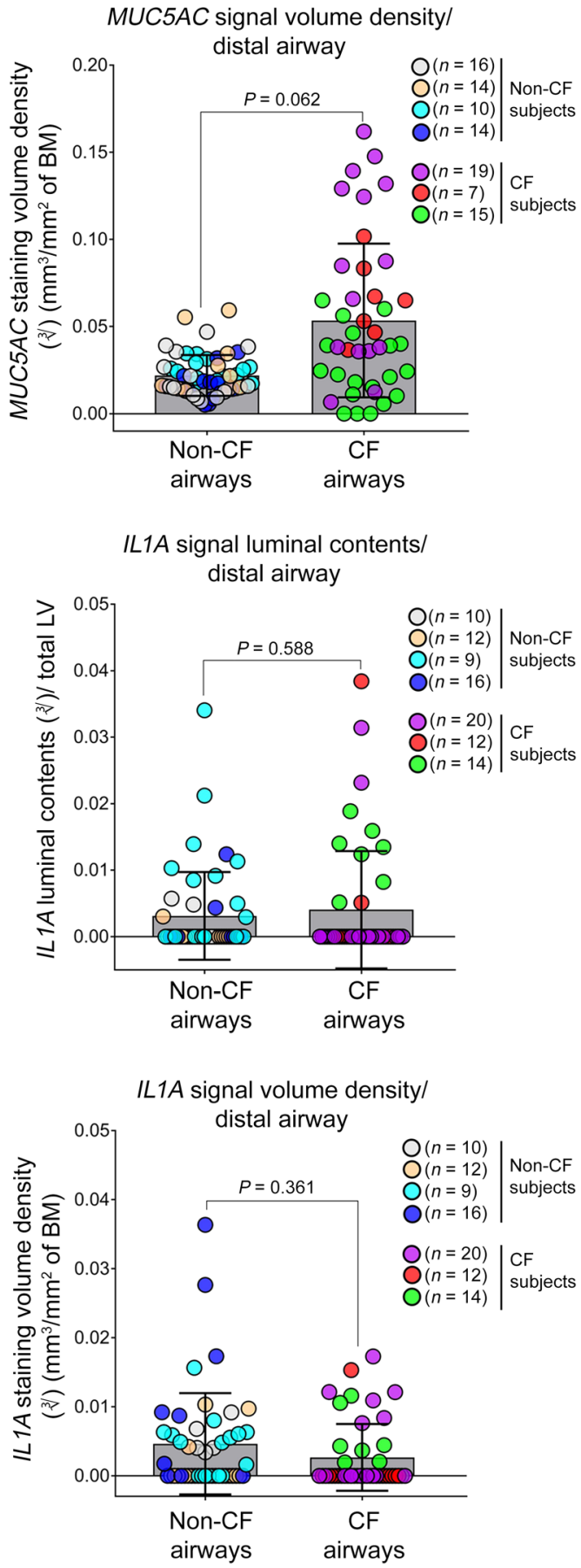

Figure 10. Quantification of MUC5B/ MUC5AC and IL1B/IL1A mRNA expression in the distal airways of control and CF lungs. (A) Quantification of the MUC5B/ MUC5AC mRNA signals in superficial epithelia lining of the distal airways (including all the airways with luminal diameter of $1.5 \mathrm{~mm}$ or less, regardless of staining status; shown in Figure 9) was determined by morphometric analysis of staining volume density. Quantification of the IL1B/IL1A mRNA signal content present in the luminal areas (B) and signal volume density in the epithelial layers (C) of the distal airways (same criteria as in A) was performed by morphometric analyses. Signal volume density in $\mathbf{A}$ and $\mathbf{C}$ was normalized to the unit surface area of the basement membrane (BM), while the luminal contents of IL $1 B / I L 1 A$ staining in $\mathbf{B}$ was normalized to the unit luminal volume (LV). Measurements of $n=4$ control subjects and $n=3$ CF subjects were performed. Individual subjects are distinguished by color-matched dots (A-C). Each color dot represents measurement from 1 airway, and the numbers of the airways used in analyses from 1 subject were annotated as $n=$ following the color dots in the figure annotations. Of note, the scatter dots/bar graphs represent cube root-transformed values. The differences of means between the 2 groups (denoted by $P$ values) were analyzed by linear mixed-effects model with subject identification number as random intercept variable. (D) mRNA expression of SPDEF, ERN2, MUC5B, and MUC5AC was quantitatively measured by TaqMan assays (normalized to endogenous CAPDH mRNA) from the airway epithelial cells freshly isolated (passage 0 ) from nonsmoker, non-CF donors (control, $n=$ 30 codes) and CF donors ( $n=24$ codes). One code means the cells obtained from 1 individual donor lung. Scatter plots present data as mean \pm SEM, and data were analyzed with 2-tailed unpaired MannWhitney $U$ test.
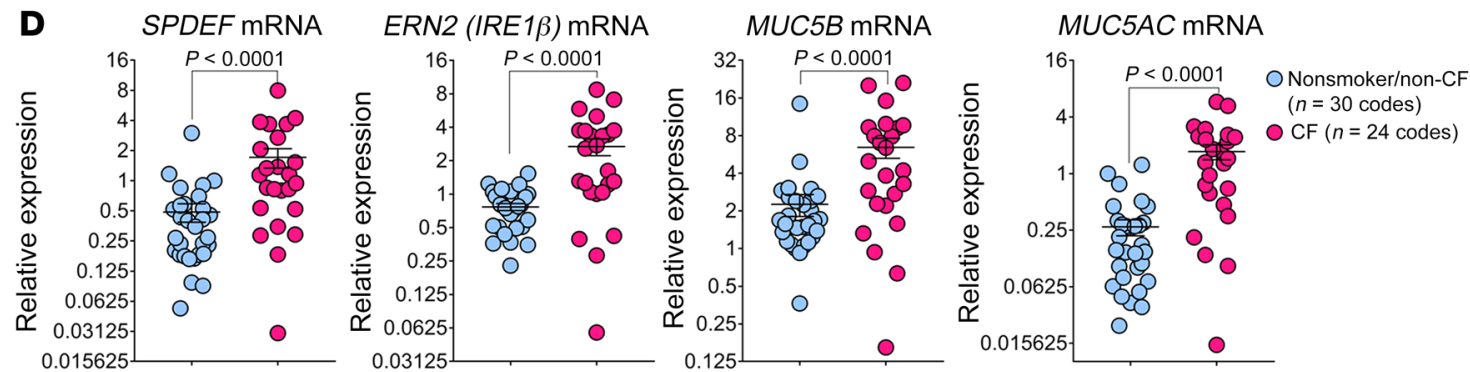
A
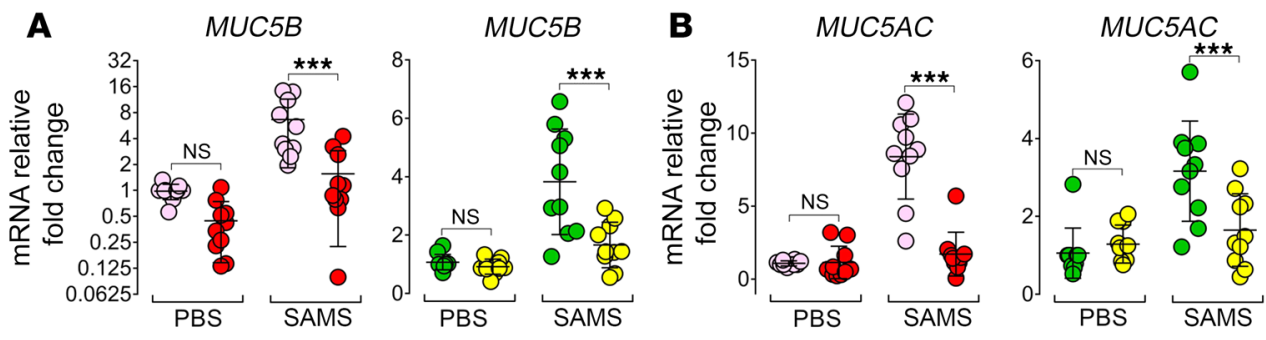

C
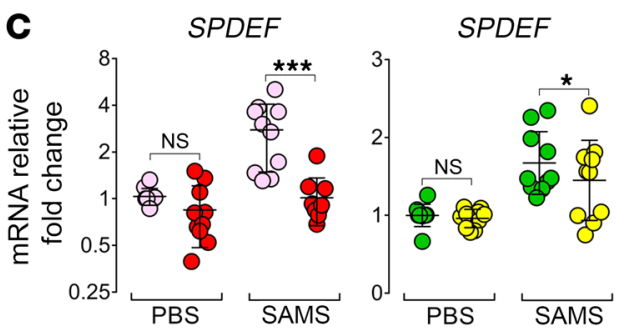

D
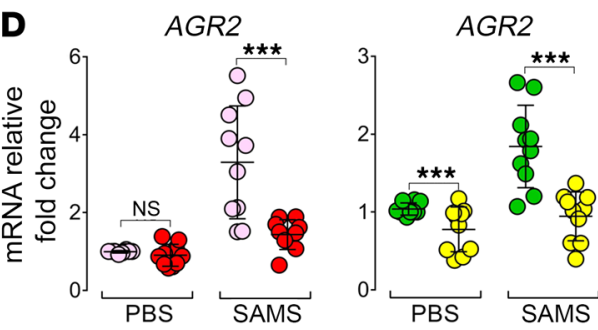

$\mathbf{E}$
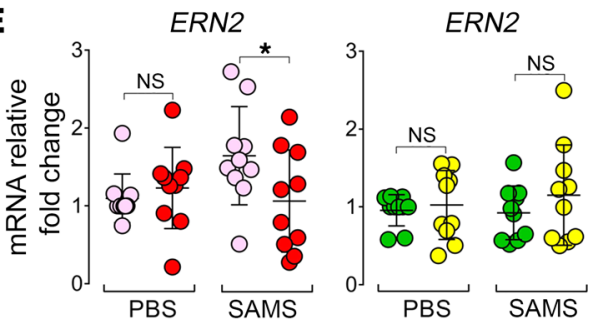

$\mathbf{F}$
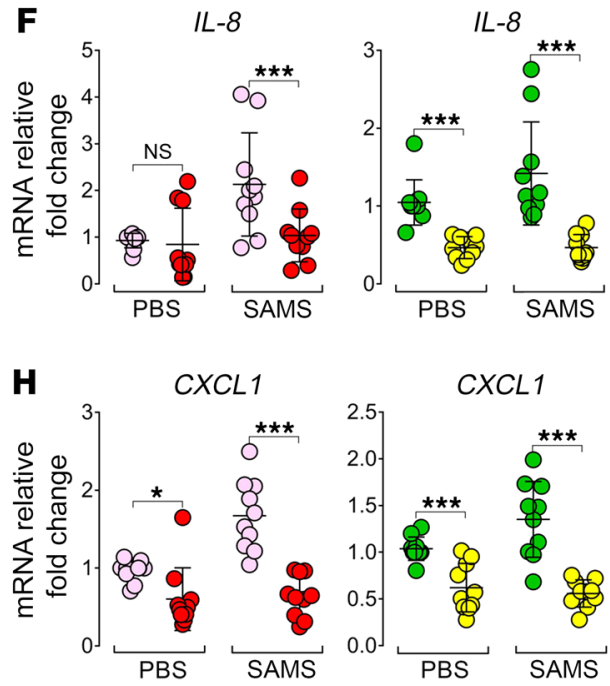

IL-6
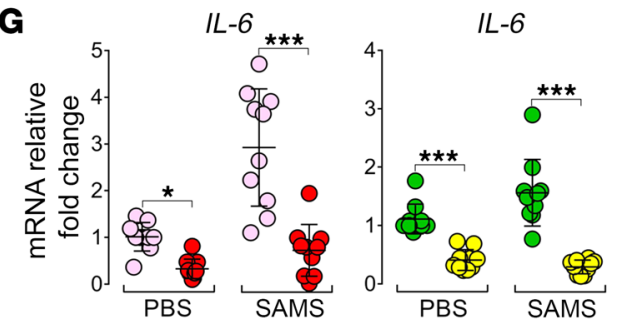

ONon-CF HBE cells + IL-1Ra

OCF HBE cells + vehicle

OCF HBE cells + IL-1Ra
Figure 11. IL-1Ra inhibits SAMS-induced expression of mucin and proinflammatory genes in both nonCF and CF HBE cells. Fully differentiated non-CF and CF HBE cells were pretreated with vehicle (PBS) or IL-1 receptor antagonist IL-1Ra (at $2 \mu \mathrm{g} /$ $\mathrm{ml}$ from the apical side and $400 \mathrm{ng} / \mathrm{ml}$ from the basolateral side) for 24 hours prior to exposure to diluted SAMS (1:40 dilution of stock SAMS with sterile PBS to reach $1 \mathrm{ng} / \mathrm{ml}$ of IL-1 $\beta$ concentration) in SAMS+IL-1Ra administration was compared with control treatment (PBS and IL-1Ra) for 3 days. mRNA expression of MUC5B (A), $\operatorname{MUC5AC}$ (B), SPDEF (C), AGR2 (D), and $E R N 2(\mathbf{E})$ and proinflammatory mediators IL 8, ILG, and CXCL1 (F-H) were quantitatively measured by TaqMan assays. Graphs present data as mean \pm SD with $n=2$ independent HBE cell cultures of non-CF (from $n=5$ donor lungs) and CF (from $n=5$ donor lungs). Data were analyzed using 2-way ANOVA followed by Tukey's test. ${ }^{*} P<0.05$; ${ }^{* *} P<0.001$, compared with vehicle-treated groups.
Previous studies investigating elucidation of the signaling pathways that mediate the persistent mucoinflammatory cycle in CF airways have reported that multiple components of airway secretions stimulate $\mathrm{CF}$ airway mucin secretion and neutrophilic inflammation $(11,13,46)$. Classic mucin secretagogues identified in SAMS include LPS, proteases, including neutrophil elastase, purine nucleotides, and a spectrum of cytokines, including IL-1 $\alpha$, IL-1 $\beta$, TNF- $\alpha$, and IL-17A/F, and neutrophilic cytokines/chemokines, including IL-8 $(13,47,48)$. It was perhaps surprising that our data indicated that there was a dominant factor in SAMS that regulated both MUC5B and MUC5AC expression, i.e., IL-1 $\alpha$ and IL-1 $\beta$, acting through the IL-1R1 (Figure 1C and Figure 11). Quantitatively, IL-1 $\beta$ was found in higher concentrations than IL-1 $\alpha$ in SAMS. IL-1 $\beta$ synthesis in the CF lung appears to be a feature of intraluminal macrophages and interstitial mononuclear cells $(49,50)$, whereas a component of epithelial release was identified for IL-1 $\alpha$ (14).

We have previously reported that both SAMS (13) and IL-1 $\beta$ (16) administration to non-CF HBE cells produce coordinated increases in mucin secretion and airway surface liquid (ASL) volume (hydration), the latter via upregulated CFTR expression/function. The CFTR-mediated fluid secretory response effectively "flushes" mucus and trapped materials from the normal lung (51). In contrast, SAMS exposure to CF HBE cells was characterized by increased mucin secretion, but no CFTR-mediated fluid secretion. Hence, CF airway epithelia responded to SAMS to produce characteristic mucus hyperconcentration (13). Importantly, IL-13, but not IL-13, mimicked the abnormal mucus hyperconcentration phenotype induced by SAMS in CF airway epithelia (Figure $4 \mathrm{C}$ and ref. 13). These differential responses likely reflect: (a) the coordination of IL-1 $\alpha$-induced and IL-1 $\beta$-induced mucin secretion and CFTR-mediated fluid secretion, the latter of which is absent in CF, versus (b) IL-13 regulation of ion channels that promote fluid secretion via the ion channel independent of CFTR, e.g., SLC26A9 (52), and calcium-activated chloride channel TMEM16A (ref. 53 and Supplemental Figure 5C).

MUC5B is the dominant secreted airway mucin in health and indeed CF $(54,55)$, and our data suggest that the increase of 
A
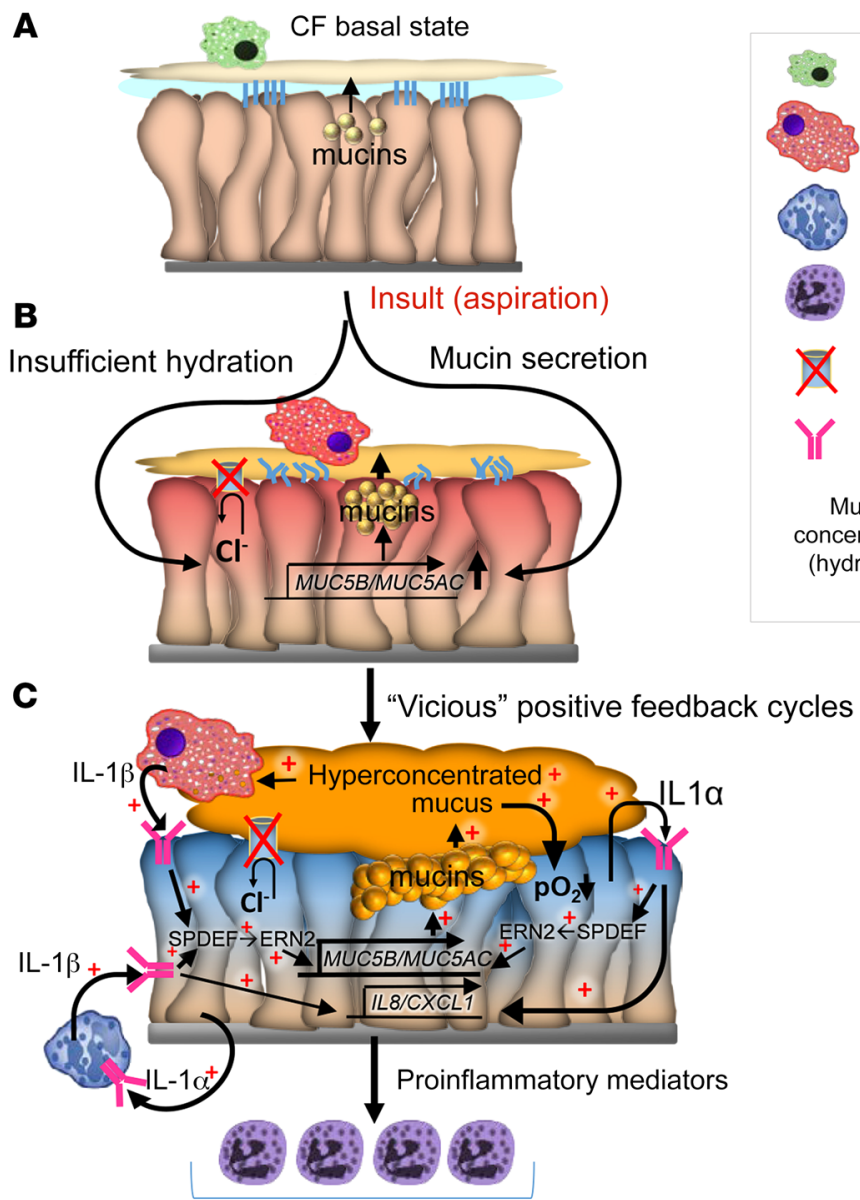

Persistent inflammation

Figure 12. Positive feedback cycle between IL-1 cytokines/inflammation and mucus hyperconcentration in the CF airways. (A) CF babies are born with sterile lungs. At their basal state, the ASL hydration and mucus concentration may be relatively normal. (B) Insults, e.g., aspiration (bile acids), promote mucin secretion, and due to loss of CFTR function, which limits $\mathrm{Cl}^{-} /$water secretion into the ASL, the mucus becomes dehydrated. Dehydrated mucus activates local luminal macrophages and produces hypoxia in epithelia. (C) Hypoxia-induced necrotic epithelia secrete IL-1 $\alpha$ (upper right and bottom left). In response to hyperconcentrated mucus or epithelial-released IL-1 $\alpha$, the luminal resident macrophages and myeloid cells in mucosa are then activated to release IL-1 $\beta$ (upper left and bottom left, respectively). Secreted IL-1 $\alpha$ and IL-1 $\beta$ activate IL-1R1 on apical or basolateral side of the epithelia to (a) induce SPDEF and ERN2-mediated mucin transcription and secretion and (b) increase expression of proinflammatory mediators, e.g., IL-8 and CXCL1. Prior to bacterial infection occurrence in CF infants/young children, the dehydrated secreted mucin exacerbates existing mucus hyperconcentration on CF airways that further activates the luminal macrophages leading to a "vicious" positive feedback cycle. Note, each step of this positive feedback cycle is denoted by a red cross. Secretion of proinflammatory mediators induces a parallel polymorphonuclear neutrophil-mediated inflammation, which persists in early CF lungs. Elastase secreted by neutrophils also activates mucin production by CF epithelia, which further contributes to mucus hyperconcentration and obstruction in the distal airways, another positive feedback cycle (not shown). $\mathrm{pO}_{2}$, partial pressure of oxygen.

in modulating MUC5AC overexpression that characterizes the CF lung. These data include the following: (a) IL- $1 \alpha$ and IL-1 $\beta$ induced MUC5AC expression to levels comparable to those with IL-13 (Figure 1, Figure 4, A and B, and Supplemental Figure 4C); (b) IL- $1 \alpha$ and IL- $1 \beta$ produced the coordinate upregulation of both MUC5AC and MUC5B mRNAs, consistent with RNAscope data from CF lungs (Figure 9A and Figure 10), whereas IL-13 raised MUC5AC, but suppressed $M U C 5 B$ mRNA levels in human airway epithelia; and (c) CRISPR/Cas9 deletion of IL-1R1 or IL-1Ra administration markedly reduced stimulation of MUC5AC expression by SAMS (Figure 1C and Figure 11).

The investigation of the role of TH2 cytokines in CF was complicated by the discrepancy in IL-13 regulation of mucin gene expression, particularly $M U C 5 B$, between human and mouse models (58). In human HBE cells, IL-13 induced MUC5AC and inhibited MUC5B mRNAs, consistent with reports of increased MUC5AC and decreased MUC5B, protein, and mRNA in sputum (59) and bronchial epithelia (60) from severe/exacerbating asthma patients, respectively. However, IL-13 induced Muc5ac without affecting $M u c 5 b$ mRNAs in $\mathrm{MTEC}$ cultures (Supplemental Figure 7A). Further, IL-13 administration to WT mice in vivo induced both Muc5b and Muc5ac protein and mRNAs (Figure 5). The in vivo effect of IL-13 on Muc 5 b levels could in part reflect IL-13-induced stimulation of Muc5b-active cytokines after intratracheal instillation, e.g., increased endogenous $I L-1 \alpha$ and $I L-1 \beta$ expression in the lung (Supplemental Figure 6B). However, the absence of Muc5b suppression by IL-13 in MTEC in vitro

MUC5B expression in airways of CF largely reflects IL-1 $\alpha$-mediated and IL-1 $\beta$-mediated regulation. However, MUC5AC is also upregulated in CF airway superficial airway epithelia (Figure 9A). MUC5AC upregulation in lung disease has typically been associated with TH2 cytokines, e.g., IL-13 (27). TH2 cytokines may play a role in $\mathrm{CF}$ mucoobstructive lung disease via an overlap "asthma-CF" scenario, i.e., $40 \%-70 \%$ of CF subjects exhibit signs of asthma (56) and $10 \%$ of CF patients have features of allergic bronchopulmonary aspergillosis (ABPA) (57). Our data, however, suggest a lesser role for TH2 signaling compared with IL- $1 \alpha$ and IL-1 $\beta$ suggests more basic differences in IL-13/MUC5B regulatory pathways in mouse versus human airway epithelia.

IL- $1 \alpha$ and IL- $1 \beta$ have been reported to be elevated in BAL from children in the Australian Respiratory Early Surveillance Team for Cystic Fibrosis (AREST-CF) study (12), concurrent with elevated mucin levels, elevated neutrophil levels, and often the absence of bacterial infection $(3,8)$. The concept of persistent, sterile, mucoinflammation that emerged from the AREST-CF studies has been reinforced by studies of neonatal Scnn $1 b-\mathrm{Tg}$ mice (airway-targeted overexpression of the epithelial $\mathrm{Na}^{+}$channel $\beta$ subunit) raised under 
gnotobiotic conditions (61) and CFTR-knockout ferrets raised with broad-spectrum antibiotics (9). Our data suggest that this "vicious" cycle of mucin secretion and inflammation in the absence or presence of bacterial infection reflects parallel positive feedback cycles. This model positions IL- $1 \alpha$ and IL- $1 \beta$ as signals that perpetuate the mucus hyperconcentration/obstruction resulting from CFTR deficiency-induced ASL depletion (Figure 12).

One limb of the positive feedback cycle describes mucus obstruction-induced epithelial hypoxia and necrosis, with IL-1 $\alpha$ release from necrotic epithelia (14). The released IL-1 $\alpha$ stimulates mucin but not CFTR-mediated fluid secretion, worsening mucus hyperconcentration and stimulating increased IL-1 $\alpha$ release. The second limb of the pathway focuses on intraluminal macrophages trapped in mucus plaques/plugs. Activation of these macrophages, likely by mucin concentration-dependent Siglec receptor activation and/or endogenous danger-signaling molecules (e.g., HMGB1) from necrotic epithelial cells $(62,63)$, stimulates secretion of IL-1 $\beta$ in a NLRP3-inflammasome-dependent manner (49, $64)$. The released IL-1 $\beta$ from luminal resident macrophages and mucosal myeloid cells (65) promotes mucin hypersecretion that, in the absence of CFTR-dependent fluid secretion, again worsens mucus hyperconcentration and obstruction, macrophage activation, and IL- $\beta$ release rates. These positive-feedback loops can be amplified by the following: (a) chronic infection (bacterial, fungal, and viral) that increases both IL- $1 \alpha$ and IL- $1 \beta$ release, as reported in BAL of CF children (12); and (b) IL- $1 \alpha$ and IL-1 $\beta$ and hypoxic cell stimulation/release of neutrophil chemokines, neutrophil accumulation, and release of neutrophil products, e.g., elastase, that may further stimulate mucin secretion (66).

Downstream signaling components of the IL-1 receptor, e.g., SPDEF and ERN2, were identified (Figure 11, C and E). Induction of SPDEF mRNA expression by SAMS or IL-1 $\beta$ (Supplemental Figure 8) provides an explanation for the role of SPDEF in non-TH2 inflammatory pulmonary diseases $(23,24,67)$. We also found that SPDEF regulated ERN2 (Figure 8 and Supplemental Figure 10 ), suggesting that SPDEF regulates mucin production, at least in part, via the UPR pathway (24). Thus, both SPDEF and ERN2 contribute to the positive feedback cycle connecting inflammatory mediators, e.g., IL- $1 \alpha$ and IL- $1 \beta$, to mucin secretion.

Blocking IL-1R1 activation by IL-1Ra (anakinra) has been tested for effects on neutrophilic inflammation and infection in CF mouse models $\left(\mathrm{Cftr}^{--}\right)$(64) and a mucoobstructive CF-like mouse model (Scnn1b-Tg) (14). Our finding that IL-1R1 was required for SAMS-, IL-1 $\alpha-$, and IL-1 $\beta$-induced, but not IL-13-triggered, mucin production by human and mouse airway epithelia (Figure 1C and Figures 6 and 11) provides further proof of concept that IL-1Ra inhibition may be therapeutically beneficial for mucus hypersecretion in CF lungs. Notably, IL-1Ra blocked SAMS-induced mucin (MUC5AC, MUC5B) and proinflammatory mediator (IL8, IL6, and CXCL1) gene expression in non-CF and CF HBE cells (Figure 11). These effects of IL-1Ra in human airway epithelia are consistent with the reduced mucus obstruction and neutrophilic inflammation/KC expression in anakinra-treated Scnn1b-Tg mice and $\mathrm{Cftr}^{-/}$mice exposed to Pseudomonas aeruginosa $(14,64)$. The cumulative data suggest that IL-1Ra may disrupt the positive feedback cycle described in Figure 12 by the following: (a) blocking the IL- $1 \alpha$ and/or IL-1 $\beta$ activation of IL-1R1, limiting mucin hypersecretion, hyperconcentration, and cellular hypoxia; and (b) reducing IL-1 $\alpha$ and/or IL-1 $\beta$ stimulation of neutrophilic inflammation, with its concomitant downstream promucin secretory activity (Figure 12 and refs. 46, 47).

Anakinra has been used for treatment of rheumatoid arthritis in humans and has a substantial record of safety. With respect to preclinical studies in CF-like lung disease, anakinra treatment did not aggravate acute $P$. aeruginosa (PAO1) infection in either WT or Scnn1b-Tg mice (68), supporting the concept that anakinra could serve as a therapeutic approach for resolving mucoinflammatory obstruction, without worsening bacterial infection in CF lung. Nevertheless, anakinra treatment was recently found to produce increased susceptibility of infection with group A Streptococcus (69), a common CF pathogen, in human subjects. In part, this susceptibility may reflect the fact that IL-1Ra inhibits SAMS-induced antimicrobial and innate defense gene expression, e.g., $\beta$-defensin and lactotransferrin, in HBE cells (Supplemental Figure 14). One interpretation of the risk-benefit aspects of anakinra suggests that this agent may have therapeutic benefits in CF subjects prior to occurrence of infection to suppress mucus obstruction, inflammation, and lung structural damage during early childhood, but may not suitable for treatment of CF patients who exhibit chronic bacterial infections. Clinical data in $\mathrm{CF}$ patients will be required to resolve this issue.

There are several limitations to this current study. First, the role of IL-1 signaling in the SMGs could not be evaluated in our model. Previous reports have suggested that IL- $1 \alpha$ and IL-1 $\beta$ are constitutively expressed in SMGs (70), where MUC5B is basally produced, and an important future question will be whether IL-1 signaling plays a role in SMG hypertrophy in CF. Second, SAMS is complex, with multiple secretagogues, and the work reported here primarily investigated IL-1 signaling in isolation. Even though we show a major role for IL-1 signaling, clearly other mediators are present, which may contribute to, or interact with, IL- $1 \alpha / \mathrm{IL}-1 \beta$ signaling. Future studies will be required to quantitate the relative effectiveness of IL- $1 \alpha$ and IL-1 $\beta$, as compared with other promucin stimulatory cytokines (e.g., IL-17A, TNF- $\alpha$ ). Third, in our studies, a significant increase in mucus concentration of apical secretions was not observed between $\mathrm{CF}$ and non-CF HBE cells at baseline in our studies. We speculate that this result reflects a relative small difference in percentage of solids at baseline between CF and non-CF HBE cells under "noninsulted", i.e., unstimulated conditions, and the variability of the mucus percentage of solids values/measurements in the HBE cells obtained from different donors. Importantly however, our results with IL-1 $\beta$ present mimic the results in CF subjects, in whom CF sputum (produced in an inflammatory environment) exhibits consistently higher concentration than sputum obtained from non-CF sputum (55).

In summary, IL- $1 \alpha$ and IL-1 $\beta$ have been identified as the dominant non-TH2 inflammatory cytokines in CF airway secretions that promote goblet cell differentiation, mucus secretion, and mucin hyperconcentration. IL- $1 \alpha$ and IL- $1 \beta$ trigger these responses to produce positive feedback cycles that maintain or worsen the CF airway mucoinflammatory state. IL-1Ra suppresses SAMSinduced mucin and proinflammatory gene expression, providing a rationale for use of IL-1Ra as a therapeutic option for treating mucus obstruction and neutrophilic inflammation-mediated airway structural damage in young CF children. 


\section{Methods}

Mouse models. WT C57BL/6J and $I l 1 \mathrm{r}^{-/-}$mice were purchased from the The Jackson Laboratory (stock no. 000664, 003245). Spdef ${ }^{-1}$ (C57BL/6N) mice were obtained from Jeffrey Whitsett's laboratory (Cincinnati Children's Hospital Medical Center, Cincinnati, Ohio, USA). Mice were housed in individually ventilated microisolator cages in a specific pathogen-free facility at the University of North Carolina at Chapel Hill. Mice were fed with regular chow and given water ad libitum on a 12-hour light/12-hour dark cycle. Mice studied were littermates when possible, age matched, and of both sexes, unless otherwise stated in the figure legends.

RNA-Seq gene-expression analysis. RNA-Seq libraries were prepared using the TruSeq RNA Sample Preparation Kit, volume 2, following the manufacturer's Low-Throughput protocol (Illumina). The libraries were sequenced on Illumina HiSeq2500 machines by the University of North Carolina Functional Genomics Core. RNA-Seq data in FASTQ format were checked for quality using the FastQC tool (https://www.bioinformatics.babraham.ac.uk/projects/fastqc). The sequence reads in FASTQ files were mapped to human genome reference assembly hg38 and Gencode.v21 gene and transcript annotation using TopHat2 (71), and expression at gene level was quantified using Cufflinks (72). The expression values in fragments per kilobase per million mapped reads (or FPKM) were filtered by mean greater than 1 among all samples and further normalized by quantile normalization, before $\log _{2}$ transformation. The $\log 2$ normalized expression data were used as input for differential gene-expression analysis using the Bioconductor package LIMMA (73), with a linear model of gene expression where the response variable of gene expression is dependent on a linear combination of cell code plus IL- $1 \beta$ treatment. Human TF gene annotations were downloaded from the AnimalTFDB database (74), current as of $12 / 2014$, and differentially expressed TFs at a $P$ value of less than 0.01 were used in hierarchical clustering analysis and heatmap generation using the Bioconductor package Complex Heatmap (75). All RNA-Seq data sets were deposited in the NCBI's Genomes Sequence Read Archive database (SRA PRJNA553230).

Statistics. To analyze morphometry data where multiple measurements from the same individuals were made (e.g., as shown in Figure 10), linear mixed-effects models were used with the R package nlme (https://cran.r-project.org/web/packages/nlme/), where the response variable is predicted from the fixed effect of interest (between different groups) and the random intercept variable of individuals. The associated $P$ values for the fixed-effect variable of interest were estimated from a $t$ distribution $(76,77)$. Statistical tests other than morphometry analysis in this manuscript were performed by Prism 7 software (Graphpad). Student's $t$ test (2 tailed, paired or unpaired, parametric or nonparametric) was used for comparison of statistical differences between 2 groups. One-way ANOVA was used to compare 3 or more groups, while 2-way ANOVA was used for comparing 2 or more groups containing 2 independent variables (e.g., HBE cell codes and infected lentiviruses). $P$ values of less than 0.05 were considered statistically significant. Data are presented as mean \pm SD or SEM unless otherwise indicated in the figure legend.

Study approval. Four deidentified CF samples from lung transplant explants and 4 control lung tissue samples from donors with no preexisting pulmonary diseases, fresh or frozen primary HBE cells from non-CF and CF donors that were used for in vitro culture and used for gene expression assays (immediately lysed after fresh isolation from the airways for total RNA purification at passage 0), and UNCN3T cells (35) (at passage 11) were provided by the Tissue Procurement and Cell Culture Core of Marsico Lung Institute University of North Carolina at Chapel Hill (ID 03-1396). Mice were maintained and studied under protocols approved by the University of North Carolina IACUC (ID: 15-249.0).

Further information is available in the Supplemental Methods.

\section{Author contributions}

GC conceived strategies, designed and carried out experiments, analyzed and interpreted results, and wrote the manuscript. LS, TK, KO, MBM, AA, JML, RCG, BDB, YKO, ASV, HD, and YD carried out experiments and reviewed the manuscript. CMPR, SHR, $\mathrm{BB}, \mathrm{MK}$, and $\mathrm{ALB}$ provided reagents and consultation and reviewed and commented on the manuscript. WKO and RCB supervised the project, interpreted results, and revised the manuscript.

\section{Acknowledgments}

This work is supported by National Heart, Lung, and Blood Institute grants P01 HL110873, P50 HL060280, P50 HL107168, P50 HL084934, UH2/UH3 HL123645, and P01 HL108808, NIDDK P30 DK065988, R01HL110906, R01HL103940, R01 HL125280, Cystic Fibrosis Foundation grants BOUCHE15R0, CFF R026-CR11, C HEN18G0 (to GC) and a New Horizons grant (to KO), Cystic Fibrosis Research Inc. The authors also thank the laboratory of Jeffrey A. Whitsett at Cincinnati Children's Hospital for donating the Spdef ${ }^{/-}$mice necessary for in vivo study, the members of the Marsico Lung Institute Tissue Procurement and Cell Culture Core for providing HBE cells and lung tissues from non-CF and CF donors and UNCN3T cells, the members of the Marsico Lung Institute Molecular Biology Core, Kristy A. Terrell for assistance with mouse genotyping, Lisa C. Morton for performing molecular cloning, Boucher lab undergraduate student Satoko Nakano for performing gene-expression assays, Kimberlie A. Burns of the Histological Core for tissue processing and performing $\mathrm{H} \& \mathrm{E}$ and AB-PAS staining, Michael Chua for assistance with microscopy, and Eric C. Roe for editorial assistance.

Address correspondence to: Richard C. Boucher, Marsico Lung Institute, Cystic Fibrosis and Pulmonary Research Center, University of North Carolina at Chapel Hill, CB\#7248, Chapel Hill, North Carolina 27599, USA. Phone: 919.966.7064; Email: richard_boucher@med.unc.edu.

\footnotetext{
1. Abman SH, Ogle JW, Butler-Simon N, Rumack $\mathrm{CM}$, Accurso FJ. Role of respiratory syncytial virus in early hospitalizations for respiratory distress of young infants with cystic fibrosis.

JPediatr. 1988;113(5):826-830.

2. Tarran R, et al. Normal and cystic fibrosis airway
}

surface liquid homeostasis. The effects of phasic shear stress and viral infections. J Biol Chem. 2005;280(42):35751-35759.

3. Esther CR, et al. Mucus accumulation in the lungs precedes structural changes and infection in children with cystic fibrosis. Sci Transl Med. 2019;11(486):eaav3488.

4. Montgomery ST, et al. Interleukin-1 is associated with inflammation and structural lung disease in young children with cystic fibrosis. J Cyst Fibros. 2018;17(6):715-722.

5 . Boucher RC. New concepts of the pathogene- 
sis of cystic fibrosis lung disease. Eur Respir J. 2004;23(1):146-158.

6. Muhlebach MS, et al. Initial acquisition and succession of the cystic fibrosis lung microbiome is associated with disease progression in infants and preschool children. PLoS Pathog. 2018;14(1):e1006798.

7. Wainwright CE, et al. Effect of bronchoalveolar lavage-directed therapy on Pseudomonas aeruginosa infection and structural lung injury in children with cystic fibrosis: a randomized trial. JAMA. 2011;306(2):163-171.

8. Montgomery ST, Mall MA, Kicic A, Stick SM, Arest CF. Hypoxia and sterile inflammation in cystic fibrosis airways: mechanisms and potential therapies. Eur Respir J. 2017;49(1):1600903.

9. Rosen $\mathrm{BH}$, et al. Infection is not required for mucoinflammatory lung disease in CFTR-knockout ferrets. Am J Respir Crit Care Med. 2018;197(10):1308-1318.

10. Bonfield TL, et al. Inflammatory cytokines in cystic fibrosis lungs. Am J Respir Crit Care Med. 1995;152(6 Pt 1):2111-2118.

11. Ribeiro CM, et al. Chronic airway infection/ inflammation induces a Ca2+i-dependent hyperinflammatory response in human cystic fibrosis airway epithelia. J Biol Chem. 2005;280(18):17798-17806.

12. Montgomery ST, et al. Interleukin-1 is associated with inflammation and structural lung disease in young children with cystic fibrosis. J Cyst Fibros. 2018;17(6):715-722.

13. Abdullah LH, et al. Mucin production and hydration responses to mucopurulent materials in normal versus cystic fibrosis airway epithelia. Am J Respir Crit Care Med. 2018;197(4):481-491.

14. Fritzsching $B$, et al. Hypoxic epithelial necrosis triggers neutrophilic inflammation via IL-1 receptor signaling in cystic fibrosis lung disease. $\mathrm{Am} \mathrm{J}$ Respir Crit Care Med. 2015;191(8):902-913.

15. Mall M, Grubb BR, Harkema JR, O'Neal WK, Boucher RC. Increased airway epithelial $\mathrm{Na}+$ absorption produces cystic fibrosis-like lung disease in mice. Nat Med. 2004;10(5):487-493.

16. Gray T, et al. Regulation of MUC5AC mucin secretion and airway surface liquid metabolism by IL-1beta in human bronchial epithelia. Am JPhysiol Lung Cell Mol Physiol. 2004;286(2):L320-L330.

17. Fujisawa T, Velichko S, Thai P, Hung LY, Huang F, Wu R. Regulation of airway MUC5AC expression by IL-1beta and IL-17A; the NF-kappaB paradigm. J Immunol. 2009;183(10):6236-6243.

18. Fujisawa T, et al. NF- $\mathrm{kB}$ mediates IL-1 $\beta$ - and IL-17A-induced MUC5B expression in airway epithelial cells. Am J Respir Cell Mol Biol. 2011;45(2):246-252.

19. Dower SK, et al. The cell surface receptors for interleukin-1 alpha and interleukin- 1 beta are identical. Nature. 1986;324(6094):266-268.

20. Arend WP. Interleukin 1 receptor antagonist. A new member of the interleukin 1 family. JClin Invest. 1991;88(5):1445-1451.

21. Chen $\mathrm{G}$, et al. Role of Spdef in the regulation of Muc5b expression in the airways of naive and mucoobstructed mice. Am J Respir Cell Mol Biol. 2018;59(3):383-396.

22. Park KS, et al. SPDEF regulates goblet cell hyperplasia in the airway epithelium. JClin Invest.
2007;117(4):978-988.

23. Chen $\mathrm{G}$, et al. SPDEF is required for mouse pulmonary goblet cell differentiation and regulates a network of genes associated with mucus production. JClin Invest. 2009;119(10):2914-2924.

24. Chen G, et al. XBP1S regulates MUC5B in a promoter variant-dependent pathway in idiopathic pulmonary fibrosis airway epithelia. Am J Respir Crit Care Med.2019;200(2):220-234.

25. Martino MB, et al. The ER stress transducer IRE1 $\beta$ is required for airway epithelial mucin production. Mucosal Immunol. 2013;6(3):639-654.

26. Tiringer K, et al. A Th17- and Th2-skewed cytokine profile in cystic fibrosis lungs represents a potential risk factor for Pseudomonas aeruginosa infection. Am J Respir Crit Care Med. 2013;187(6):621-629.

27. Bonser LR, Zlock L, Finkbeiner W, Erle DJ. Epithelial tethering of MUC5AC-rich mucus impairs mucociliary transport in asthma. J Clin Invest. 2016;126(6):2367-2371.

28. Lachowicz-Scroggins ME, et al. Corticosteroid and long-acting $ß$-agonist therapy reduces epithelial goblet cell metaplasia. Clin Exp Allergy. 2017;47(12):1534-1545.

29. Martinovich KM, et al. Conditionally reprogrammed primary airway epithelial cells maintain morphology, lineage and disease specific functional characteristics. Sci Rep. 2017;7(1):17971.

30. Gentzsch M, et al. Pharmacological rescue of conditionally reprogrammed cystic fibrosis bronchial epithelial cells. Am J Respir Cell Mol Biol. 2017;56(5):568-574.

31. Suprynowicz FA, et al. Conditionally reprogrammed cells represent a stem-like state of adult epithelial cells. Proc Natl Acad Sci U S A. 2012;109(49):20035-20040.

32. Rock JR, et al. Transmembrane protein $16 \mathrm{~A}$ (TMEM16A) is a $\mathrm{Ca}^{2+}$-regulated $\mathrm{Cl}^{-}$secretory channel in mouse airways. J Biol Chem. 2009;284(22):14875-14880.

33. Rajavelu P, Chen G, Xu Y, Kitzmiller JA, Korfhagen TR, Whitsett JA. Airway epithelial SPDEF integrates goblet cell differentiation and pulmonary Th2 inflammation. J Clin Invest. 2015;125(5):2021-2031.

34. Calfon M, et al. IRE1 couples endoplasmic reticulum load to secretory capacity by processing the XBP-1 mRNA. Nature. 2002;415(6867):92-96.

35. Fulcher ML, et al. Novel human bronchial epithelial cell lines for cystic fibrosis research. Am JPhysiol Lung Cell Mol Physiol. 2009;296(1):L82-L91.

36. Schroeder BW, et al. AGR2 is induced in asth$\mathrm{ma}$ and promotes allergen-induced mucin overproduction. Am J Respir Cell Mol Biol. 2012;47(2):178-185.

37. Vareille M, Kieninger E, Edwards MR, Regamey $\mathrm{N}$. The airway epithelium: soldier in the fight against respiratory viruses. Clin Microbiol Rev. 2011;24(1):210-229.

38. Boon M, et al. Morphometric analysis of explant lungs in cystic fibrosis. Am J Respir Crit Care Med. 2016;193(5):516-526.

39. Boucher RC. Evidence for airway surface dehydration as the initiating event in CF airway disease. J Intern Med. 2007;261(1):5-16.

40. Jorth $P$, et al. Direct lung sampling indicates that established pathogens dominate early infections in children with cystic fibrosis. Cell Rep. 2019;27(4):1190-1204.e3.

41. Pillarisetti $\mathrm{N}$, et al. Infection, inflammation, and lung function decline in infants with cystic fibrosis. Am J Respir Crit Care Med. 2011;184(1):75-81.

42. Shah VS, et al. Airway acidification initiates host defense abnormalities in cystic fibrosis mice. Science. 2016;351(6272):503-507.

43. Hill DB, et al. Pathological mucus and impaired mucus clearance in cystic fibrosis patients result from increased concentration, not altered $\mathrm{pH}$. Eur Respir J. 2018;52(6):1801297.

44. Button B, et al. Roles of mucus adhesion and cohesion in cough clearance. Proc Natl Acad Sci US A. 2018;115(49):12501-12506.

45. Hoegger MJ, et al. Impaired mucus detachment disrupts mucociliary transport in a piglet model of cystic fibrosis. Science. 2014;345(6198):818-822.

46. Voynow JA, Young LR, Wang Y, Horger T, Rose MC, Fischer BM. Neutrophil elastase increases MUC5AC mRNA and protein expression in respiratory epithelial cells. Am J Physiol. 1999;276(5):L835-L843.

47. Gehrig S, et al. Lack of neutrophil elastase reduces inflammation, mucus hypersecretion, and emphysema, but not mucus obstruction, in mice with cystic fibrosis-like lung disease. Am J Respir Crit Care Med. 2014;189(9):1082-1092.

48. Sagel SD, Chmiel JF, Konstan MW. Sputum biomarkers of inflammation in cystic fibrosis lung disease. Proc Am Thorac Soc. 2007;4(4):406-417.

49. Tang A, et al. Inflammasome-mediated IL-1 $\beta$ production in humans with cystic fibrosis. PLOS ONE. 2012;7(5):e37689.

50. Folco EJ, Sukhova GK, Quillard T, Libby P. Moderate hypoxia potentiates interleukin- $1 \beta$ production in activated human macrophages. Circ Res. 2014;115(10):875-883.

51. Luan X, et al. Cystic fibrosis swine fail to secrete airway surface liquid in response to inhalation of pathogens. Nat Commun. 2017;8(1):786.

52. Anagnostopoulou P, et al. SLC26A9-mediated chloride secretion prevents mucus obstruction in airway inflammation. J Clin Invest. 2012;122(10):3629-3634.

53. Huang F, et al. Calcium-activated chloride channel TMEM16A modulates mucin secretion and airway smooth muscle contraction. Proc Natl Acad Sci U S A. 2012;109(40):16354-16359.

54. Okuda K, et al. Localization of secretory mucins MUC5AC and MUC5B in normal/healthy human airways. Am J Respir Crit Care Med 2019;199(6):715-727.

55. Henderson AG, et al. Cystic fibrosis airway secretions exhibit mucin hyperconcentration and increased osmotic pressure. JClin Invest. 2014;124(7):3047-3060.

56. McCuaig S, Martin JG. How the airway smooth muscle in cystic fibrosis reacts in proinflammatory conditions: implications for airway hyperresponsiveness and asthma in cystic fibrosis. Lancet Respir Med. 2013;1(2):137-147.

57. Laufer $\mathrm{P}$, et al. Allergic bronchopulmonary aspergillosis in cystic fibrosis. J Allergy Clin Immunol. 1984;73(1 Pt 1):44-48.

58. Bonser LR, Erle DJ. Airway mucus and asthma: the role of MUC5AC and MUC5B.JClin Med. 
2017;6(12):E112.

59. Lachowicz-Scroggins ME, et al. Abnormalities in MUC5AC and MUC5B protein in airway mucus in asthma. Am J Respir Crit Care Med. 2016;194(10):1296-1299.

60. Woodruff PG, et al. T-helper type 2-driven inflammation defines major subphenotypes of asthma. Am J Respir Crit Care Med. 2009;180(5):388-395.

61. Livraghi-Butrico A, et al. Mucus clearance, MyD88-dependent and MyD88-independent immunity modulate lung susceptibility to spontaneous bacterial infection and inflammation. Mucosal Immunol. 2012;5(4):397-408.

62. Zhang X, Mosser DM. Macrophage activation by endogenous danger signals. J Pathol. 2008;214(2):161-178.

63. Janssen WJ, Stefanski AL, Bochner BS, Evans CM. Control of lung defence by mucins and macrophages: ancient defence mechanisms with modern functions. Eur Respir J. 2016;48(4):1201-1214.

64. Iannitti RG, et al. IL-1 receptor antagonist ameliorates inflammasome-dependent inflammation in murine and human cystic fibrosis. Nat Commun.
2016;7:10791.

65. Di Paolo NC, Shayakhmetov DM. Interleukin $1 \alpha$ and the inflammatory process. Nat Immunol. 2016;17(8):906-913.

66. Voynow JA, et al. Neutrophil elastase induces mucus cell metaplasia in mouse lung. Am J Physiol Lung Cell Mol Physiol. 2004;287(6):L1293-L1302.

67. Xu Y, et al. Single-cell RNA sequencing identifies diverse roles of epithelial cells in idiopathic pulmonary fibrosis. JCI Insight. 2016;1(20):e90558.

68. Schütte A, et al. The effect of the antiinflammatory IL-1R antagonist anakinra in mice with CF-like lung disease and Pseudomonas aeruginosa infection. Pneumologie. 2016;70(7):P27.

69. LaRock CN, et al. IL-1 $\beta$ is an innate immune sensor of microbial proteolysis. Sci Immunol. 2016;1(2):eaah3539.

70. Cox RA, Burke AS, Traber DL, Herndon DN, Hawkins HK. Production of pro-inflammatory polypeptides by airway mucous glands and its potential significance. Pulm Pharmacol Ther. 2007;20(2):172-177.

71. Kim D, Pertea G, Trapnell C, Pimentel H, Kelley R, Salzberg SL. TopHat2: accurate alignment of transcriptomes in the presence of insertions, deletions and gene fusions. Genome Biol. 2013;14(4):R36.

72. Trapnell C, et al. Differential gene and transcript expression analysis of RNA-Seq experiments with TopHat and Cufflinks. Nat Protoc. 2012;7(3):562-578.

73. Ritchie ME, et al. limma powers differential expression analyses for RNA-Sequencing and microarray studies. Nucleic Acids Res. 2015;43(7):e47.

74. Zhang HM, et al. AnimalTFDB: a comprehensive animal transcription factor database. Nucleic Acids Res. 2012;40(Database issue):D144-D149.

75. Gu Z, Eils R, Schlesner M. Complex heatmaps reveal patterns and correlations in multidimensional genomic data. Bioinformatics. 2016;32(18):2847-2849.

76. Pinheiro JC, Bates DM. Unconstrained parametrizations for variance-covariance matrices. Statistics and Computing. 1996;6(3):289-296.

77. Pinheiro JC, Bates DM. Mixed-Effects Models in S and S-PLUS. New York, New York, USA: SpringerVerlag; 2000. 